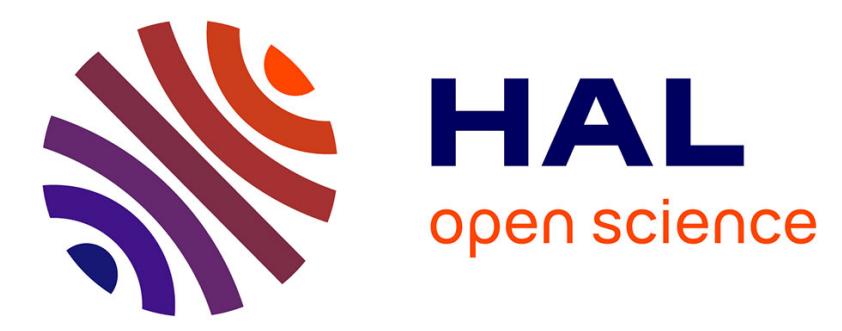

\title{
On 3D DDFV discretization of gradient and divergence operators. I. Meshing, operators and discrete duality.
}

Boris Andreianov, Mostafa Bendahmane, Florence Hubert, Stella Krell

\section{To cite this version:}

Boris Andreianov, Mostafa Bendahmane, Florence Hubert, Stella Krell. On 3D DDFV discretization of gradient and divergence operators. I. Meshing, operators and discrete duality.. IMA Journal of Numerical Analysis, 2012, 32 (4), pp.1574-1603. 10.1093/imanum/drr046 . hal-00355212v3

\section{HAL Id: hal-00355212 \\ https://hal.science/hal-00355212v3}

Submitted on 11 Mar 2011

HAL is a multi-disciplinary open access archive for the deposit and dissemination of scientific research documents, whether they are published or not. The documents may come from teaching and research institutions in France or abroad, or from public or private research centers.
L'archive ouverte pluridisciplinaire HAL, est destinée au dépôt et à la diffusion de documents scientifiques de niveau recherche, publiés ou non, émanant des établissements d'enseignement et de recherche français ou étrangers, des laboratoires publics ou privés.

\section{(c) (1) $\$$}

Distributed under a Creative Commons Attribution - NonCommerciall 4.0 International 


\title{
ON 3D DDFV DISCRETIZATION \\ OF GRADIENT AND DIVERGENCE OPERATORS. I. MESHING, OPERATORS AND DISCRETE DUALITY.
}

\author{
B. ANDREIANOV, M. BENDAHMANE, F. HUBERT, AND S. KRELL
}

\begin{abstract}
This work is intended to provide a convenient tool for the mathematical analysis of a particular kind of finite volume approximation which can be used, for instance, in the context of nonlinear and/or anisotropic diffusion operators in 3D. Following the so-called DDFV (Disctere Duality Finite Volume) approach developed by F. Hermeline and by K. Domelevo and $\mathrm{P}$. Omnès in $2 \mathrm{D}$, we consider a "double" covering $\mathfrak{T}$ of a three-dimensional domain by a rather general primal mesh and by a well-chosen "dual" mesh. The associated discrete divergence operator $\operatorname{div}^{\mathfrak{T}}$ is obtained by the standard finite volume approach. A simple and consistent discrete gradient operator $\nabla^{\mathfrak{T}}$ is defined by local affine interpolation that takes into account the geometry of the double mesh. Under mild geometrical constraints on the choice of the dual volumes, we show that $-\operatorname{div}^{\mathfrak{I}}, \nabla^{\mathfrak{T}}$ are linked by the "discrete duality property", which is an analogue of the integration-by-parts formula. The primal mesh need not be conformal, and its interfaces can be general polygons.

We give several numerical examples for anisotropic linear diffusion problems; good convergence properties are observed. The sequel [3] of this paper will summarize some key discrete functional analysis tools for DDFV schemes and give applications to proving convergence of DDFV schemes for several nonlinear degenerate parabolic PDEs.
\end{abstract}

\section{Contents}

1. Introduction

1.1. The context of DDFV and related schemes 2

1.2. The goal of the paper 4

1.3. Brief description of the operators 4

1.4. Outline of the paper 5

2. Double meshes and the associated gradient and divergence operators 6

2.1. Construction of "double" meshes 6

2.2. Spaces of discrete functions and fields, discrete divergence and gradient operators 8

2.3. Cartesian DDFV meshes in 3D 9

3. The discrete duality property 10

4. On the choice of the face centers $x_{K \mid L}$ and other generalizations 13

5. Numerical experiments for linear diffusion problems 16

Appendix: A reconstruction property in the plane 20

References $\quad 22$

Date: March 11, 2011.

2000 Mathematics Subject Classification. 65N12,65M12.

Key words and phrases. Finite volume approximation, Gradient reconstruction, Discrete gradient, Discrete duality, 3D CeVe-DDFV, Consistency, Anisotropic elliptic problems, General mesh, Non-conformal mesh.

A large part of this paper was completed during the visit of the first author to the University of Concepción, Chile, supported by the FONDECYT program No.7080187. The authors thank François Hermeline and Charles Pierre for discussions and remarks. 


\section{INTRODUCTION}

1.1. The context of DDFV and related schemes. "Discrete duality" finite volume (DDFV) discretization of linear and nonlinear diffusion operators, introduced for the Laplace problem in 2D by Hermeline in [44] and by Domelevo and Omnès in [30] (some key ideas appear already in the works of Nicolaïdes [56] and Nicolaïdes and $\mathrm{Hu}[48,56]$ ), is one of possible discretization strategies which applies to very general meshes and to a large variety of PDEs including the Stokes problem, the Maxwell equations, nonlinear and linear anisotropic diffusion and convectiondiffusion problems (see e.g. [5, 9, 13, 15, 22, 25, 28, 29, 30, 43, 44, 45, 46, 48, 49, 50, 56, 57] and references therein). The name "DDFV" stresses one important aspect of this $2 \mathrm{D}$ scheme, namely the duality between the discrete gradient and the discrete divergence operators in use. Yet this property is shared by various numerical schemes (e.g. the mimetic ones, for which the discrete duality property underlies the definition of the scheme); actually, the name DDFV refers also to the strategy of using "double" meshes in $2 \mathrm{D}$ with both cell and vertex unknowns.

Among different discretization approaches intended to resolve the difficulties coming either from anisotropy/nonlinearity of the PDE under consideration or from the need of working on non-orthogonal, non-conformal, locally refined meshes, let us mention those of $[1,2,7,16,17,27$, $31,32,33,34,35,36,37,38,39,40,52,53,59]$; this list is by no means exhaustive. Several of the above works present dimension-independent constructions, others are specific to $2 \mathrm{D}$ and their extension to 3D requires new ideas (this is the case of the DDFV schemes). A 2D Benchmark that reflects the behaviour of some of the above methods on linear diffusion problems was presented by Herbin and Hubert in [41].

Among the aforementioned works, the closest to the DDFV strategy is the "complementary volumes" strategy (Walkington [59]) as described by Handlovičová, Mikula and Sgallari in [40], that was introduced in $2 \mathrm{D}$ in [54] (see also $[55,39]$ ). The same idea was used in $2 \mathrm{D}$, in slightly different ways, in [2] and in [10] (see also [4, Sec.2.1]). A 3D version was formulated in [18] and analyzed in [39].

From a different viewpoint, the DDFV methodology of [30] is inspired by the gradient reconstruction strategy "per diamond" introduced by Coudière, Vila and Villedieu [27].

In contrast to the above complementary volumes methods, a 2D DDFV mesh is a "double" mesh. It consists of a primal mesh, which in general can be non-orthogonal and non-conformal (e.g., locally refined), and of an appropriately chosen "dual" mesh. A discrete function is then a superposition of two constant per volume functions, one on the primal mesh and the other one on the dual mesh. From the primal and the dual mesh, a "diamond mesh" is generated (a more general point of view is suggested in $[9, \S I X . B])$; the "gradient" of a discrete function is reconstructed as a constant per diamond vector field. Convergence analysis techniques for these 2D schemes are well developed by now (see e.g. [9, 13, 30] for details).

In $3 \mathrm{D}$, several types of methods inspired by the 2D DDFV methodology were already proposed. They differ by the number and the interpretation of their unknowns and/or by the construction of the additional control volumes. We have recently learned of another interpretation, developed by Pierre [58], in which the DDFV methods are seen as standard finite volume methods associated to only one mesh.

(A) The construction due to Pierre (see [57] and [24, 26, 23]) involves, like in the $2 D$ case, unknowns at both centers and vertices of the initial (primal) mesh. The faces of the primal mesh are either triangles or quadrangles. The dual mesh used by Pierre et al. is rather unusual: it recovers the domain twice. The discrete duality property does hold for this construction (see [57, 26]), making it a DDFV method.

From the practical point of view, the method of [57] was successfully applied to discretization of the elliptic-parabolic bidomain cardiac system in [24, 25, 26].

(B) Two constructions generalizing the 2D case are due to Hermeline. The one of [46] provides a very wide family of finite volume schemes using various kinds of 3D "double" meshes. In this approach, additional unknowns are added at the faces of the primal mesh; they are linked to unknowns at the vertices through interpolation in order to overcome the 
difficulties of gradient reconstruction. No study of duality features was conducted for this method and, except in some particular situations, the discrete duality fails.

From the practical point of view, this method showed good convergence properties on several tests including mild heterogeneity and anisotropy, see [46]; but it was supplanted by the more constrained method [47] of the same author where the face unknown is eventually eliminated. Numerically, the method of [47] improves over the one of [46], e.g., on strongly distorted meshes. The improved method of Hermeline does enjoy the discrete duality property, see [47, Th.1]; and it is presented with specific adaptation to the more delicate case of discontinuous diffusion coefficients (cf. [13] for the 2D case).

In [47], the faces of the initial mesh are either triangles or quadrangles, and the face centers are barycenters. These restrictions can be bypassed. Indeed, the method of [47] actually enters the framework $(\mathrm{C})$ below $^{1}$ when the diffusion tensor is continuous.

(C) The construction of Karlsen and the first two authors [4,5] differs from the one of (A) by the form of the dual cells, which is more conventional (see Sections 2,4 for details). Yet (A) and (C) lead to the same matrices on linear diffusion problems (see [23]); only the projection operators differ and this induces slightly different discretization of the source term. The improved construction of Hermeline [47] that took over the method (B) actually amounts to the same method $(\mathrm{C})$.

The focus in [5] was on the orthogonal (Delaunay-Voronoï, according to [46, 47]) meshes with triangular faces, while the gradient reconstruction announced in [4] is applicable for the general case. In the present paper, we describe this approach for a wide class of meshes (no restriction on number of face vertices appears; neither orthogonality nor conformity is needed) and, most importantly, we assess the essential discrete duality feature of the DDFV method, for this wide class of meshes.

From the practical point of view, the method $(\mathrm{C})$ was used in [6] for a further numerical study of the bidomain cardiac model.

(D) The last scheme developed by Coudière and the third author in $[19,20]$ modified the idea of [46] and added unknowns not only at faces, but also at the edges of the primal mesh. These unknowns used for gradient reconstruction are not interpolated, contrarily to [46]. Both face and edge points are seen as the cell centers for a new mesh which does not appear in the previous constructions. Consequently, in the finite volume approximation of $[19,20]$ three meshes are involved, making it a "triple" mesh. The discrete duality property for this construction was shown in [20]. The approach of [20] recently gave rise to the scheme on 3D primal meshes with quadrangular faces, as sketched in [38, Sec. 2.2]; this is a $3 \mathrm{D}$ analogue of the $2 \mathrm{D}$ construction in [9, IX.B]. Let us point out that the scheme of [38, Sec. 2.2] can be rather naturally re-interpreted as a DDFV scheme of kind (A).

From the practical point of view, the DDFV method (D) was successfully applied in [20] (for Leray-Lions elliptic problems or general meshes) and in [51] (for the Stokes problem).

Numerical results of all these schemes $[24,46,47,20,6]$ show good convergence properties, even on strongly distorted meshes. A direct comparison of these schemes will be held through the 3D anisotropic benchmark [11, 21, 23] and [42].

According to the location of unknowns wrt the primal mesh, one can qualify the methods (A), (B) in the version [47], and (C), as CeVe-DDFV methods (cell and vertex unknowns); and the method (D), as a CeVeFE-DDFV method (cell, vertex and face+edge unknowns). We have already pointed out (cf. [6, 23]) that discretizations originating form (A),(B) (version of [47]) and $(\mathrm{C})$ coincide, up to details that are not of prime importance. While (D) appears as a quite different method (in particular, the data structure used for implementation is different), a strong connection exists when the primal meshes have quadrangular faces. Indeed, the method (A) can be put in correspondence with the version [38] of the method (D) (upon splitting the dual mesh

\footnotetext{
${ }^{1}$ The meshes and discrete divergence of [46] coincide (up to unnecessary restrictions) with those of our paper; thus the discrete gradients also coincide, since the discrete duality property that holds for both schemes. Yet the motivations behind the reconstruction of the discrete gradient of [47] (see also [57]) and the ours are rather different.
} 
of (A) into two meshes each of them recovering the domain only once). Further, the method (C) on cartesian meshes (see Section 2.3) rewrites as a variant of (D), see the proof of [3, Prop. 3.7].

1.2. The goal of the paper. This paper gives a detailed account on the CeVe-DDFV scheme in its version $(C)$, on general meshes, and it prepares the ground for the sequel [3] where we provide convenient tools for the mathematical analysis of DDFV discretizations on three-dimensional domains. We exhibit a consistent discrete gradient operator which possesses the same kind of "integration-by-parts" property as the 3D schemes of [57], [5], [20] or as the 2D DDFV schemes (see $[9,30])$. This means that the discrete gradient operator $\nabla^{\mathfrak{T}}$ is dual to the usual finite volume discrete divergence operator $-\operatorname{div}^{\mathfrak{T}}$ on the double mesh, for appropriately defined $L^{2}$ pairings of discrete functions and of discrete vector fields. A brief summary of our 3D DDFV construction and of the discrete duality result, along with an application on Delaunay-Voronoï meshes detailed in [5], were given in [4] by Karlsen and the first two authors; with respect to [4], the present paper gives a simpler (but equivalent) formula for the discrete gradient, many generalizations, examples and numerical tests.

The reason we focus on the discrete duality features is that they make the finite volume discretization of numerous elliptic operators "structure-preserving"; and they underlie the convergence proofs (see, e.g., [30, 9, 13, 5, 20, 6] and the sequel [3] of this paper).

Note that different variants of the "discrete duality property" hold for the schemes of [32, 31], of [33, 34, 37, 35], of [7]. For the "complementary finite volumes" schemes as described in [40, 2, 10, 4], a discrete duality property, completely similar to the one shown in the present paper, is true in 2D (see [4] and Remark 4.6). For the mimetic finite difference (MFD) schemes, the discrete duality is built into the definition of the schemes (so that the MFD schemes can be defined through only one of the two discrete operators). Also note that the discrete duality property can be somewhat relaxed. Eymard, Herbin and Guichard introduced in [38] the notion of "gradient scheme", including some 3D DDFV schemes. "Gradient schemes" according to [38] fulfill an approximate discrete duality property, property that is sufficient to infer convergence at least for the linear test cases as those considered in Section 5 of the present paper (see [38, Sec.2]).

1.3. Brief description of the operators. In the 3D DDFV framework (C) we postulate, independently one from another ${ }^{2}$, quite natural definitions for both $\nabla^{\mathfrak{T}}$ (of a discrete field $\overrightarrow{\mathcal{F}}$ ) and $-\operatorname{div}^{\mathfrak{T}}$ (of a discrete function $u^{\mathfrak{T}}$ ) on a double mesh $\mathfrak{T}$. Namely (up to notational details; cf. Section 2.2),

- $-\operatorname{div}^{\mathfrak{T}} \overrightarrow{\mathcal{F}}$ is obtained by the Green-Gauss formula from the integration of $-\overrightarrow{\mathcal{F}} \cdot \vec{n}$ on the boundaries of the volumes of the double mesh (this is the core of any finite volume approach);

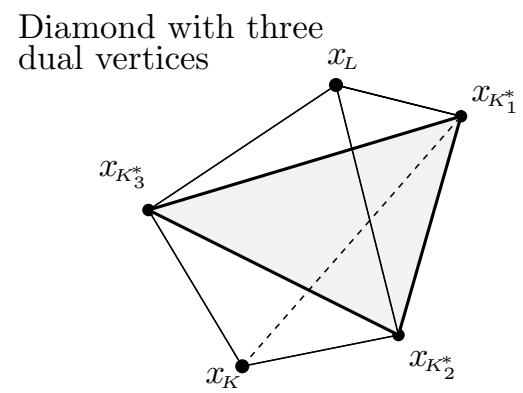

Figure 1. (a) A diamond: the simplest case

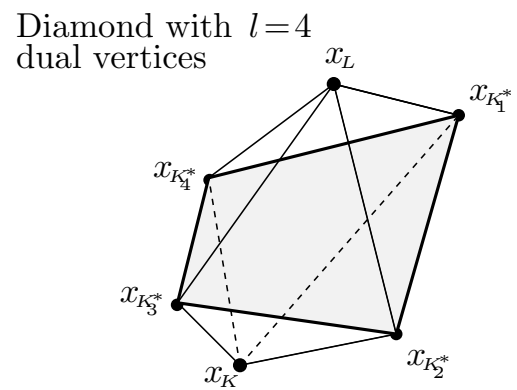

(b) The general case (here, $l=4$ )

\footnotetext{
2 at this point, our approach differs in its spirit from the one of mimetic finite difference (MFD) schemes
} 
- $\nabla^{\mathfrak{T}} u^{\mathfrak{I}}$ is reconstructed piecewise constant per diamond, by a well-chosen ponderation of the vertex values; the reconstruction is consistent with affine functions.

Consider a generic diamond $D=D^{K, L}$ built on two "primal vertices" $x_{K}, x_{L}$ and on $l$ "dual vertices" $x_{K_{i}^{*}}, i=1, \ldots, l$ of the double mesh (see Figure 1). In this case, the vector $\vec{g}=: \nabla_{D} u^{\mathfrak{\tau}}$ is assembled from its projection $\operatorname{Proj}_{D} \vec{g}$ on the direction $\overrightarrow{x_{K} x_{L}}$ and from its projection $\operatorname{Proj}_{D}^{*} \vec{g}$ on the plane containing the face $K \mid L$. Each of the projections is uniquely determined from the values of $u^{\mathfrak{T}}$ at the vertices of $D$, as follows.

First, the projection $\operatorname{Proj}_{D} \vec{g}$ is obtained from the values $u_{K}, u_{L}$ at the points $x_{K}, x_{L}$ in a straightforward way:

$$
\operatorname{Proj}_{D} \nabla_{D} u^{\mathfrak{\tau}}=\frac{u_{L}-u_{K}}{\left|\overrightarrow{x_{K} x_{L}}\right|} \overrightarrow{x_{K} x_{L}}
$$

Second, the projection $\operatorname{Proj}_{D}^{*} \vec{g}$ is reconstructed from the values $x_{K_{i}^{*}}$ attached to the points $x_{K_{i}^{*}}$ in the face $K L$. If $l=3$ (see Figure 1(a)), there exists a unique affine function $w$ on the face $K \mid L$ such that $w\left(x_{K_{i}^{*}}\right)=u_{K_{i}^{*}}$, for $i=1,2,3$. Naturally, we impose that $\operatorname{Proj}_{D}^{*} \vec{g}=\nabla w$. Thanks to a remarkable identity (Lemma 5.1 and Corollary 5.2 given in Appendix A), this can be rewritten as follows (with $l=3$ and $l+1:=1$ ):

$$
\operatorname{Proj}_{D}^{*} \nabla_{D} u^{\mathfrak{T}}=\frac{1}{m_{K \mid L}} \sum_{i=1}^{l}\left(u_{K_{i+1}^{*}}-u_{K_{i}^{*}}\right)\left[\vec{n} \times \overline{x_{K \mid L} x_{K_{i}^{*} \mid K_{i+1}^{*}}}\right],
$$

where $m_{K \mid L}$ is the area of the face $K \mid L, \vec{n}_{K \mid L}$ is the unit normal vector to $K \mid L$ pointing from $K$ to $L, x_{K \mid L}$ is some fixed point (a center) in the face $K \mid L$, and $x_{K_{i}^{*} \mid K_{i+1}^{*}}$ is the middlepoint (the center) of the edge $\left[x_{K_{i}^{*}}, x_{K_{i+1}^{*}}\right]$.

Further, when a diamond spans $l>3$ dual vertices $x_{K_{1}^{*}}, \ldots, x_{K_{l}^{*}}$ (see Figure 1(b)), an affine function $w$ interpolating the vertex values does not exist in general. Yet formula (1) still makes sense; moreover, by Lemma 5.1 and Corollary 5.2, its right-hand side still yields $\nabla w$ whenever $w$ exists. Due to this remarkable identity ${ }^{3}$, we postulate the choice (1) to define $\operatorname{Proj}_{D}^{*} \nabla_{D} u^{\mathcal{T}}$. The justification of the remarkable identity is the most technical part of the construction, and it is of independent interest; we postpone it to Appendix A.

To give an example, 3D DDFV schemes on cartesian meshes are described in Section 2.3, and the associated discrete operators are written down explicitly.

1.4. Outline of the paper. Along with the construction itself, the key theoretical results of the paper are those of Proposition 2.3 and Proposition 3.2. The link between consistency and discrete duality is based upon the "reconstruction property" of Lemma 5.1 (Appendix A) which is of independent interest.

For simplicity, in Section 2 we restrict our attention to the case of convex primal volumes, and impose constraints on the choice of the "centers" of volumes and faces; these constraints can be relaxed (see Remarks 4.1, 4.2 and Example 4.3 in Section 4 devoted to generalizations).

The proof of the discrete duality property given in Section 3 requires a rather involved notation, including a convention on the orientation of the diamonds. The notation is introduced in Section 2 and in Lemma 3.3, and illustrated with the help of figures. One benefit from the notation is that we are able to give closed-form formulas for the discrete gradient and for the discrete divergence in terms of vector products and mixed products in $\mathbb{R}^{3}$.

Let us point out that the notation in terms of discrete operators, discrete functions and fields and their scalar products as introduced in Section 2 is also aimed to guide the reader through the convergence proofs presented in the sequel [3] of this paper. This notation stresses the far-reaching analogy between the continuous framework and the discrete DDFV framework.

While convergence proofs and some numerical experiments for several degenerate parabolic problems will be briefly presented in [3], numerical tests on linear anisotropic diffusion problems are the object of Section 5. These tests are based on the $3 D$ benchmark for linear anisotropic

\footnotetext{
${ }^{3}$ It is an analogue of the celebrated "magical formula" of [32, Lemma 6.1].
} 
diffusion problems prepared by R. Herbin and the third author, [42]. Convergence of the method in these test cases is easily established using the discrete duality property and the tools of [30, 9, 13, 3].

\section{Double MeShes AND THE ASSOCIATED GRADIENT AND DIVERGENCE OPERATORS}

Let $\Omega$ be a polyhedral open bounded subset of $\mathbb{R}^{3}$. In what follows, we introduce the notation related to "double" finite volume schemes; each piece of new notation is given in italic script. The notation is redundant in many cases, which is convenient because the role of objects we introduce is often multifold. Most of the notations are illustrated with the help of figures.

Throughout the paper, $\|\vec{a}\|$ denotes the euclidean norm of $\vec{a} \in \mathbb{R}^{3} ; \vec{a} \cdot \vec{b}$ (respectively, $\vec{a} \times \vec{b}$ ) denotes the scalar product (respectively, the vector product) of $\vec{a}, \vec{b} \in \mathbb{R}^{3} ;\langle\vec{a}, \vec{b}, \vec{c}\rangle$ denotes the mixed product of $\vec{a}, \vec{b}, \vec{c} \in \mathbb{R}^{3}$. We use extensively the geometric meaning of these products.

2.1. Construction of "double" meshes.

- A partition of $\Omega$ is a finite set of disjoint open polyhedral subsets of $\Omega$ such that $\Omega$ is contained in their union, up to a set of zero three-dimensional measure.

A "double" finite volume mesh of $\Omega$ is a triple $\mathfrak{T}=\left(\overline{\mathfrak{M}^{0}}, \overline{\mathfrak{M}^{*}}, \mathfrak{D}\right)$ described below.

- We take $\mathfrak{M}^{\circ}$ a partition of $\Omega$ into open polyhedra. We assume them convex.

Each $K \in \mathfrak{M}^{\circ}$ is called control volume and supplied with an arbitrarily chosen center $x_{K}$; for simplicity, we assume $x_{K} \in K$. We call $\partial \mathfrak{M}^{\circ}$ the set of all faces of control volumes that are included in $\partial \Omega$. These faces are considered as boundary control volumes; for $K \in \partial \mathfrak{M}^{\circ}$, we choose a center $x_{K} \in K$. We denote by $\overline{\mathfrak{M}^{o}}$ the union $\mathfrak{M}^{o} \cup \partial \mathfrak{M}^{o}$. We call vertex (of $\mathfrak{M}^{o}$ ) any vertex of any control volume $K \in \mathfrak{M}^{o}$.

- In the case of conformal meshes, we call neighbours of $K$, all control volumes $L \in \overline{\mathfrak{M}^{o}}$ such that $K$ and $L$ have a common face. In the non-conformal case, it is enough that $\partial_{K}$ and $\partial L$ intersect along a set of non-zero two-dimensional measure.

The set of all neighbours of $K$ is denoted by $\mathcal{N}(K)$. Note that if $L \in \mathcal{N}(K)$, then $K \in \mathcal{N}(L)$; in this case we simply say that $K$ and $L$ are (a couple of) neighbours.

If $K$ and $L$ are neighbours, we denote by $K L$ the interface (face) (or its part, in the nonconformal case) $\partial K \cap \partial L$ that separates $K$ and $L$. Due to the convexity of $K, L$, the interface $K L$ is planar.

- A generic vertex of $\mathfrak{M}^{\circ}$ is denoted by $x_{K^{*}}$; it will be associated later with a unique dual control volume $K^{*} \in \overline{\mathfrak{M}}^{*}$. Each face $K \mid L$ is supplied with a face center $x_{K \mid L}$ which should lie in ${ }_{K} \mid L \quad$ (the more general situation is discussed in Remarks 4.1, 4.2). For two neighbour vertices $x_{K^{*}}$ and $x_{L^{*}}$ (i.e., vertices of $\mathfrak{M}^{o}$ joined by an edge of some polygon $K \mid L$ ), we denote by $x_{K^{*} \mid L^{*}}$ the middlepoint of the segment $\left[x_{K^{*}}, x_{L^{*}}\right]$.

- Now if $K \in \overline{\mathfrak{M}^{0}}$ and $L \in \mathcal{N}(K)$, assume $x_{K^{*}}, x_{L^{*}}$ are two neighbour vertices of the interface $K \mid L$. We denote by $T_{K^{*} ; L^{*}}^{K ; L}$ the tetrahedron formed by the points $x_{K}, x_{K^{*}}, x_{K \mid L}, x_{K^{*} \mid L^{*}}$.

A generic tetrahedron $T_{K^{*} ; L^{*}}^{K ; L}$ is called an element of the mesh and denoted by $T$ (see Figure 4); the set of all elements is denoted by $\mathcal{T}$. If $x_{K}$ is a vertice of $T \in \mathcal{T}$, then we say that $T$ is associated $^{4}$ with the volume $K$, and we write $T \sim K$.

- Define the volume $K^{*}$ associated with a vertice $x_{K^{*}}$ of $\mathfrak{M}^{\circ}$ as the union of all elements $T \in \mathcal{T}$ having $x_{K^{*}}$ for one of its vertices. The collection $\overline{\mathfrak{M}^{*}}$ of all such $K^{*}$ forms another partition of $\Omega$.

If $x_{K^{*}} \in \Omega$, we say that $K^{*}$ is a dual control volume and write $K^{*} \in \mathfrak{M}^{*}$; and if $x_{K^{*}} \in \partial \Omega$, we say that $K^{*}$ is a boundary dual control volume and write $K^{*} \in \partial \mathfrak{M}^{*}$. Thus $\overline{\mathfrak{M}^{*}}=\mathfrak{M}^{*} \cup \partial \mathfrak{M}^{*}$. We call dual vertex (of $\overline{\mathfrak{M}^{*}}$ ) any vertex of any dual control volume $K^{*} \in \overline{\mathfrak{M}^{*}}$. Note that by construction, the set of vertices coincides with the set of dual centers $x_{K^{*}}$; the set of dual vertices consists of centers $x_{K}$, face centers $x_{K \mid L}$ and edge centers (middlepoints) $x_{K^{*} \mid L^{*}}$.

\footnotetext{
${ }^{4}$ Because we have made the assumption that $x_{K \mid L} \in K \mid L$, the relation $T \sim K$ simply means that $T$ is included in $K$. We will develop a more general point of view in Remark 4.2. The same observation applies to the notation $T \sim K^{*}, T \sim D, S \sim D$ introduced later on.
} 
Picturing dual volumes in 3D is a hard task; an idea of how the dual volumes look like can be inferred from a deformation of the example given in Section 2.3 (see Figure 3).

If $x_{K^{*}}$ is a vertice of $T \in \mathcal{T}$, then we say that $T$ is associated with the volume $K^{*}$, and we write $T \sim K^{*}$.

- We denote by $\mathcal{N}^{*}\left(K^{*}\right)$ the set of (dual) neighbours of a dual control volume $K^{*}$, and by $K^{*} L^{*}$, the (dual) interface $\partial K^{*} \cap \partial L^{*}$ between dual neighbours $K^{*}$ and $L^{*}$.

- For an element $T=T_{K^{*} ; L^{*}}^{K ; L}$, we denote by $\sigma_{T}$ the face of $T$ contained within the plane $K \mid L$. The area of $\sigma_{T}$ is denoted by $m_{T}$; finally, $\vec{n}_{\mathrm{S}}$ denotes the unit normal vector to $\sigma_{T}$ exterior to $T$. Similarly, we denote by $\sigma_{T}^{*}$ the face of $T$ contained within the plane $K^{*} L^{*}$. The area of $\sigma_{T}^{*}$ is denoted by $m_{T}^{*}$, and $\vec{n}_{T}^{*}$ is the corresponding exterior unit normal vector.

- Finally, we introduce the partition of $\Omega$ into diamonds.

If $K, L \in \overline{\mathfrak{M}^{\circ}}$ are neighbours, then the union of the convex hull of $x_{K}$ and ${ }_{K} \mid L$ with the convex hull of $x_{L}$ and $K \mid L$ is called diamond and denoted by $D^{K \mid L}$. In the sequel, to each diamond we will prescribe an orientation by fixing arbitrarily the orientation of the segment $\left[x_{K}, x_{L}\right]$.

We denote by $\mathfrak{D}$ the set of all diamonds. Generic diamond in $\mathfrak{D}$ is denoted by $D$. Notice that $\mathfrak{D}$ is a partition of $\Omega$. We will write $T \sim D$ to signify that the element $T \in \mathcal{T}$ is included within $D$ (or, more generally, is associated with $D$; see Section 4).
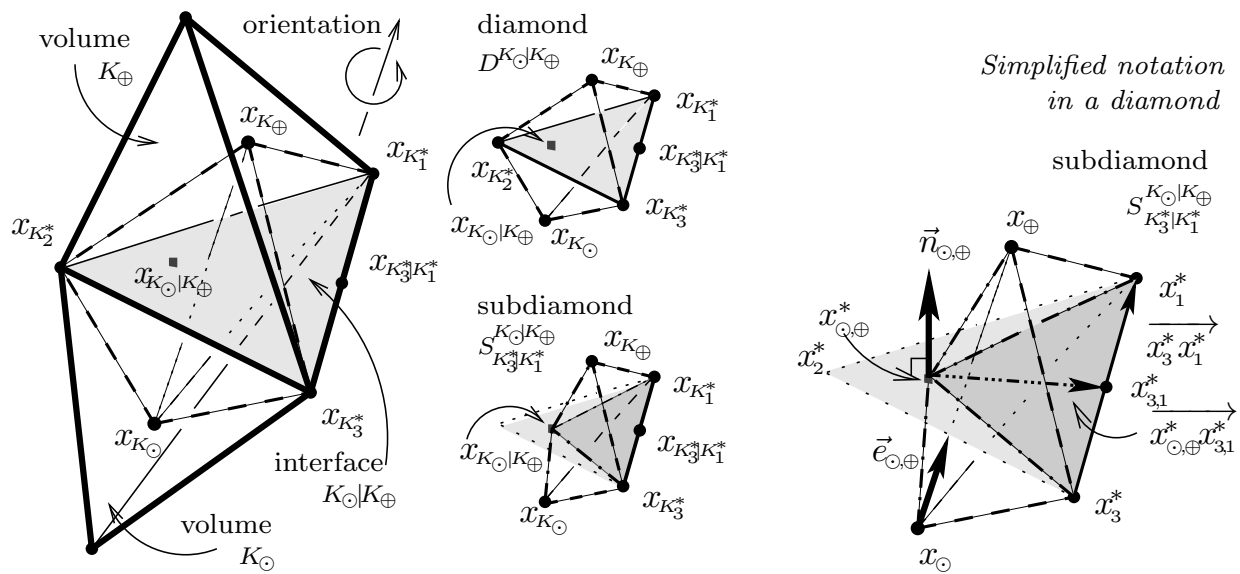

Figure 2. 3D neighbour volumes, diamond, subdiamond. Z Zoom on a subdiamond.

- (see Figure 2) Whenever the orientation of a diamond $D$ should be cared of, the primal vertices defining it will be denoted by $x_{K_{\odot}}, x_{K_{\oplus}}$ in such a way that the vector $\overrightarrow{x_{K_{\odot}} x_{K_{\oplus}}}$ has the orientation prescribed for the diamond. The oriented diamond is then denoted by $D^{K_{\odot} \mid K_{\oplus}}$. We denote by $\vec{e}_{K_{\odot}, K_{\oplus}}$ the corresponding unit vector, and by $d_{K_{\odot}, K_{\oplus}}$, the length of $\overrightarrow{x_{K_{\odot}} x_{K_{\oplus}}}$. We denote by $\vec{n}_{K_{\odot} \mid K_{\oplus}}$ the unit normal vector to $K_{\odot} \mid K_{\oplus}$ such that $\vec{n}_{K_{\odot} \mid K_{\oplus}} \cdot \vec{e}_{K_{\odot}, K_{\oplus}}>0$.

The normal vector $\vec{n}_{K_{\odot} \mid K_{\oplus}}$ to $K_{\odot} \mid K_{\oplus}$ being fixed, this induces the orientation in the corresponding face $K_{\odot} \mid K_{\oplus}$, which is a convex polygon with $l$ vertices : we denote the vertices of $K_{\odot} \mid K_{\oplus}$ by $x_{K_{i}^{*}}, i \in \llbracket 1, l \rrbracket$, enumerated in the direct sense. By convention, we assign $x_{K_{l+1}^{*}}:=x_{K_{1}^{*}}$. We denote by $\vec{e}_{K_{i}^{*}, K_{i+1}^{*}}$ the unit normal vector pointing from $x_{K_{i}^{*}}$ towards $x_{K_{i+1}^{*}}$, and by $d_{K_{i}^{*}, K_{i+1}^{*}}$, the length of $\overrightarrow{x_{K_{i}^{*}}^{*} x_{K_{i+1}^{*}}}$.

In order to lighten the notation in an oriented diamond (and in its subdiamonds, introduced in Section 3 below and pictured in Figures 2,4), we will drop the $K$ 's in the subscripts and denote the objects introduced above by $x_{\odot}, x_{\oplus}, \vec{e}_{\odot, \oplus}, d_{\odot, \oplus}, \vec{n}_{\odot, \oplus}$ and by $x_{i}^{*}, \vec{e}_{i, i+1}^{*}, d_{i, i+1}^{*}$ whenever $D^{K_{\odot} \mid K_{\oplus}}$ is fixed. We also denote by $x_{i, i+1}^{*}$ the middlepoint $x_{K_{i}^{*} \mid K_{i+1}^{*}}$ of the segment $\left[x_{i}, x_{i+1}\right]$, and by $x_{\odot, \oplus}^{*}$, the center $x_{K_{\odot} \mid K_{\oplus}}$ of $K_{\odot} \mid K_{\oplus}$.

- For a diamond $D=D^{K_{\odot} \mid K_{\oplus}}$, we denote by $\operatorname{Proj}_{D}$ the operator of orthogonal projection of $\mathbb{R}^{3}$ on the line $\left\langle\vec{e}_{K_{\odot}, K_{\oplus}}>\right.$; we denote by $\operatorname{Proj}_{D}^{*}$ the operator of orthogonal projection of $\mathbb{R}^{3}$ on the plane containing the interface $K_{\odot} \mid K_{\oplus}$. 
- We denote by $\operatorname{Vol}(A)$ the three-dimensional Lebesgue measure of $A$ which can stand for a control volume, a dual control volume, an element, a diamond, etc..

Remark 2.1. Diamonds permit to define the discrete gradient operator (see (4),(5) below). In turn, elements (and, even more conveniently, the subdiamonds introduced later on) permit to define the discrete divergence operator (see (2),(3) and (12) below).

In the context of $2 D$ "double" schemes, introducing diamonds is quite standard (see e.g. [9, 30]). Subdiamonds are "hidden" in the $2 D$ construction : they actually coincide with diamonds.

The above definitions are illustrated and generalized in Remark 4.2 at the end of the paper.

\subsection{Spaces of discrete functions and fields, discrete divergence and gradient operators.}

- A discrete function on $\Omega$ is a set $w^{\mathfrak{T}}=\left(w^{\mathfrak{M}^{\circ}}, w^{\mathfrak{M}^{*}}\right)$ consisting of two sets of real values $w^{\mathfrak{M}^{o}}=\left(w_{K}\right)_{K \in \mathfrak{M}^{o}}$ and $w^{\mathfrak{M}^{*}}=\left(w_{K^{*}}\right)_{K^{*} \in \mathfrak{M}^{*}}$. The set of all such functions is denoted by $\mathbb{R}^{\mathfrak{T}}$.

A discrete function on $\bar{\Omega}$ is a set $w^{\overline{\mathfrak{T}}}=\left(w^{\mathfrak{M}^{o}}, w^{\mathfrak{M}^{*}} ; w^{\partial \mathfrak{M}^{o}}, w^{\partial \mathfrak{M}^{*}}\right) \equiv\left(w^{\mathfrak{T}} ; w^{\partial \mathfrak{T}}\right)$ consisting of

$$
w^{\mathfrak{M}^{o}}=\left(w_{K}\right)_{K \in \mathfrak{M}^{o}}, w^{\mathfrak{M}^{*}}=\left(w_{K^{*}}\right)_{K^{*} \in \mathfrak{M}^{*}}, w^{\partial \mathfrak{M}^{o}}=\left(w_{K}\right)_{K \in \partial \mathfrak{M}^{o}}, w^{\partial \mathfrak{M}^{*}}=\left(w_{K^{*}}\right)_{K^{*} \in \partial \mathfrak{M}^{*}} .
$$

The set of all such functions is denoted by $\mathbb{R}^{\overline{\mathfrak{T}}}$. In case all the components of $w^{\partial \mathfrak{I}}=\left(w^{\partial \mathfrak{M}^{o}}, w^{\partial \mathfrak{M}^{*}}\right)$ are zero, we write $w^{\overline{\mathfrak{s}}} \in \mathbb{R}_{0}^{\overline{\mathfrak{s}}}$.

- A discrete field on $\Omega$ is a set $\overrightarrow{\mathcal{F}}^{\mathfrak{T}}=\left(\overrightarrow{\mathcal{F}}_{D}\right)_{D \in \mathcal{D}}$ of vectors of $\mathbb{R}^{3}$. The set of all discrete fields is denoted by $\left(\mathbb{R}^{3}\right)^{\mathfrak{D}}$. If $\overrightarrow{\mathcal{F}}^{\mathfrak{T}}$ is a discrete field on $\Omega$, we assign $\overrightarrow{\mathcal{F}}_{T}=\overrightarrow{\mathcal{F}}_{D}$ whenever $T \sim D$.

- On the set $\left(\mathbb{R}^{3}\right)^{\mathcal{D}}$ of discrete fields $\overrightarrow{\mathcal{F}}^{\mathfrak{T}}$, we define the operator $\operatorname{div}^{\mathfrak{T}}[\cdot]$ of discrete divergence by

$$
\operatorname{div}^{\mathfrak{T}}: \overrightarrow{\mathcal{F}}^{\mathfrak{T}} \in\left(\mathbb{R}^{3}\right)^{\mathcal{D}} \mapsto \operatorname{div}^{\mathfrak{T}} \overrightarrow{\mathcal{F}}^{\mathfrak{T}}=\left(\left(\operatorname{div}_{K} \overrightarrow{\mathcal{F}}^{\mathfrak{T}}\right)_{K \in \mathfrak{M}^{o}},\left(\operatorname{div}_{K^{*}} \overrightarrow{\mathcal{F}}^{\mathfrak{T}}\right)_{K^{*} \in \mathfrak{M}^{*}}\right) \in \mathbb{R}^{\mathfrak{T}}
$$

where the entries $\operatorname{div}_{K} \overrightarrow{\mathcal{F}}^{\mathfrak{T}}$, $\operatorname{div}_{K^{*}} \overrightarrow{\mathcal{F}}^{\mathfrak{T}}$ of the discrete function $\operatorname{div}^{\mathfrak{I}} \overrightarrow{\mathcal{F}}^{\mathfrak{T}}$ on $\Omega$ are given by

$$
\operatorname{div}_{K} \overrightarrow{\mathcal{F}}^{\mathfrak{T}}=\frac{1}{\operatorname{Vol}(K)} \sum_{T \sim K} m_{T} \overrightarrow{\mathcal{F}}_{T} \cdot \vec{n}_{T}, \quad \operatorname{div}_{K^{*}} \overrightarrow{\mathcal{F}}^{\mathfrak{T}}=\frac{1}{\operatorname{Vol}\left(K^{*}\right)} \sum_{T \sim K^{*}} m_{T}^{*} \overrightarrow{\mathcal{F}}_{T} \cdot \vec{n}_{T}^{*} .
$$

Formulas (3) correspond to the standard procedure of finite volume discretization applied on each part of the double mesh $\boldsymbol{T}$. A more explicit formula (12) for the discrete divergence is given in (12) and (15) in Section 3.

By construction, it is clear that the discrete divergence operator is consistent. Its consistency (in the weak sense; cf. [3, Prop. 3.1(iii)]) can also be inferred from the one of the discrete gradient operator (see Proposition 2.3) and from the discrete duality property that is the object of the next section.

- On the set $\mathbb{R}^{\overline{\mathfrak{T}}}$ of discrete functions $w^{\overline{\mathfrak{T}}}$ on $\bar{\Omega}$, we define the operator $\nabla^{\mathfrak{T}}[\cdot]$ of discrete gradient

$$
\nabla^{\mathfrak{T}}: w^{\overline{\mathfrak{T}}} \in \mathbb{R}^{\overline{\mathfrak{T}}} \mapsto \nabla^{\mathfrak{T}} w^{\overline{\mathfrak{T}}}=\left(\nabla_{D} w^{\overline{\mathfrak{T}}}\right)_{D \in \mathcal{D}} \in\left(\mathbb{R}^{3}\right)^{\mathfrak{D}}
$$

where the entry $\nabla_{D} w^{\overline{\mathfrak{T}}}$ of the discrete field $\nabla^{\mathfrak{T}} w^{\overline{\mathfrak{T}}}$ corresponding to $D=D^{K_{\odot} \mid K_{\oplus}}$ is given by (5)

$$
\nabla_{D} w^{\overline{\mathfrak{T}}} \text { is s.t. }\left\{\begin{array}{l}
\operatorname{Proj}_{D}\left(\nabla_{D} w^{\overline{\mathfrak{s}}}\right)=\frac{w_{\oplus}-w_{\odot}}{d_{\odot, \oplus}} \vec{e}_{\odot, \oplus}, \\
\operatorname{Proj}_{D}^{*}\left(\nabla_{D} w^{\overline{\mathfrak{s}}}\right)=\frac{2}{\sum_{i=1}^{l}\left\langle\vec{n}_{\odot, \oplus}, \overline{x_{\odot, \oplus}^{*} x_{i, i+1}^{*}}, \overline{x_{i}^{*} x_{i+1}^{*}}\right\rangle} \sum_{i=1}^{l}\left(w_{i+1}^{*}-w_{i}^{*}\right)\left[\vec{n}_{\odot, \oplus} \times \overrightarrow{x_{\odot, \oplus}^{*} x_{i, i+1}^{*}}\right] .
\end{array}\right.
$$

with $w_{\odot}=w_{K_{\odot}}, w_{\oplus}=w_{K_{\oplus}}, w_{i}^{*}=w_{K_{i}^{*}}$, etc. (we use the simplified notation in the diamond $D=D^{K_{\odot} \mid K_{\oplus}}$, as depicted in Figure 2).

Remark 2.2. In (5), the primal mesh $\overline{\mathfrak{M}^{o}}$ serves to reconstruct one component of the gradient, which is the one in the direction $\vec{e}_{\odot, \oplus}$. The dual mesh $\overline{\mathfrak{M}^{*}}$ serves to reconstruct, with the help 
of the formula (26) of Appendix A, the two other components which are the components in the plane containing $K_{\odot} \mid K_{\oplus}$.

The above choice of the discrete gradient is explained in the Introduction; the specific choice of $\operatorname{Proj}_{D}^{*}\left(\nabla_{D} w^{\overline{\mathfrak{z}}}\right)$ stems from the analysis of Appendix A. Now we point out the crucial fact that (5) and Corollary 5.2 in Appendix A imply the consistency of our gradient approximation:

Proposition 2.3. Let $w_{\odot}, w_{\oplus},\left(w_{i, i+1}^{*}\right)_{i=1}^{l}$ be the values at the points $x_{\odot}, x_{\oplus},\left(x_{i, i+1}^{*}\right)_{i=1}^{l}$, respectively, of an affine on $D=D^{K_{\odot} \mid K_{\oplus}}$ function $w$. Then $\nabla_{D} w^{\bar{\Sigma}}$ coincides with the value of $\nabla w$ on $D$.

Now let us give a more explicit representation of $\nabla_{D} w^{\bar{\Sigma}}$.

Lemma 2.4. The definition (5) of $\nabla_{D} w^{\bar{\Sigma}}$ is equivalent to the representation

$$
\nabla_{D} w^{\bar{\Sigma}}=\frac{1}{6 \operatorname{Vol}(D)} \sum_{i=1}^{l}\left\{\frac{\left\langle\overrightarrow{x_{\odot} x_{\oplus}}, \overrightarrow{x_{\odot, \oplus}^{*} x_{i, i+1}^{*}}, \overrightarrow{x_{i}^{*} x_{i+1}^{*}}\right\rangle}{\overrightarrow{x_{\odot} x_{\oplus}} \cdot \vec{n}_{\odot, \oplus}}\left(w_{\oplus}-w_{\odot}\right) \vec{n}_{\odot, \oplus}+2\left(w_{i+1}^{*}-w_{i}^{*}\right)\left[\overrightarrow{x_{\odot} x_{\oplus}} \times \overrightarrow{x_{\odot, \oplus}^{*} x_{i, i+1}^{*}}\right]\right\}
$$

Notice that in formula (6), we can substitute the mixed product $\left\langle\overrightarrow{x_{\odot} x_{\oplus}}, \overrightarrow{x_{\odot, \oplus}^{*} x_{i, i+1}^{*}}, \overrightarrow{x_{i}^{*} x_{i+1}^{*}}\right\rangle$ by $6 \operatorname{Vol}\left(S_{K_{i}^{*} \mid K_{i+1}^{*}}^{K_{\odot} \mid K_{\oplus}}\right)$, where $S_{K_{i}^{*} K_{i+1}^{*}}^{K_{\odot} \mid K_{\oplus}}$ is the subdiamond introduced later on (see Figure 4 ).

Proof. First, notice that if $\vec{p}=\operatorname{Proj}_{D}\left(\nabla_{D} w^{\bar{\Sigma}}\right), \overrightarrow{p^{*}}=\operatorname{Proj}_{D}\left(\nabla_{D} w^{\bar{\Sigma}}\right)$ are given, we have

$$
\nabla_{D} w^{\bar{\Sigma}}=\overrightarrow{p^{*}}+\frac{\vec{e}_{\odot, \oplus} \cdot\left(\vec{p}-\overrightarrow{p^{*}}\right)}{\vec{e}_{\odot, \oplus} \cdot \vec{n}_{\odot, \oplus}} \vec{n}_{\odot, \oplus}
$$

Consider the contribution of the term $\left(w_{i+1}^{*}-w_{i}^{*}\right)\left[\vec{n}_{\odot, \oplus} \times \overrightarrow{x_{\odot, \oplus}^{*} x_{i, i+1}^{*}}\right]$ in $\overrightarrow{p^{*}}$ into formula (7). It amounts to

$$
\frac{\left(w_{i+1}^{*}-w_{i}^{*}\right)}{\vec{e}_{\odot, \oplus} \cdot \vec{n}_{\odot, \oplus}}\left\{\left[\vec{n}_{\odot, \oplus} \times \overrightarrow{x_{\odot, \oplus}^{*} x_{i, i+1}^{*}}\right]\left(\vec{e}_{\odot, \oplus} \cdot \vec{n}_{\odot, \oplus}\right)-\vec{n}_{\odot, \oplus}\left(\left[\vec{n}_{\odot, \oplus} \times \overrightarrow{x_{\odot, \oplus}^{*} x_{i, i+1}^{*}}\right] \cdot \vec{e}_{\odot, \oplus}\right)\right\} .
$$

Using the well-known "BAC minus CAB" identity $(\vec{b} \cdot \vec{a}) \vec{c}-(\vec{c} \cdot \vec{a}) \vec{b}$ and the fact that $\vec{n}_{\odot, \oplus} \times$ $\left[\vec{n}_{\odot, \oplus} \times \overrightarrow{x_{\odot, \oplus}^{*} x_{i, i+1}^{*}}\right]=\overrightarrow{x_{\odot, \oplus}^{*} x_{i, i+1}^{*}}$, we transform the above expression into $\frac{\left(w_{i+1}^{*}-w_{i}^{*}\right)}{\vec{e}_{\odot, \oplus} \cdot \vec{n}_{\odot, \oplus}}\left[\vec{\odot}_{\odot, \oplus} \times \overrightarrow{x_{\odot, \oplus}^{*} x_{i, i+1}^{*}}\right]=$ $\frac{\left(w_{i+1}^{*}-w_{i}^{*}\right)}{\overrightarrow{x_{\odot} x_{\oplus} \cdot \vec{n}_{\odot, \oplus}}}\left[\overrightarrow{x_{\odot} x_{\oplus}} \times \overrightarrow{x_{\odot, \oplus}^{*} x_{i, i+1}^{*}}\right]$. Further, notice that

$$
\left(\overrightarrow{x_{\odot} x_{\oplus}} \cdot \vec{n}_{\odot, \oplus}\right)\left\langle\vec{n}_{\odot, \oplus}, \overrightarrow{x_{\odot, \oplus}^{*} x_{i, i+1}^{*}}, \overrightarrow{x_{i}^{*} x_{i+1}^{*}}\right\rangle=\left\langle\overrightarrow{x_{\odot} x_{\oplus}}, \overrightarrow{x_{\odot, \oplus}^{*} x_{i, i+1}^{*}}, \overrightarrow{x_{i}^{*} x_{i+1}^{*}}\right\rangle,
$$

and that

$$
\sum_{i=1}^{l}\left\langle\overrightarrow{x_{\odot} x_{\oplus}}, \overrightarrow{x_{\odot, \oplus}^{*} x_{i, i+1}^{*}}, \overrightarrow{x_{i}^{*} x_{i+1}^{*}}\right\rangle=6 \operatorname{Vol}(D) .
$$

Finally, the contribution of $\vec{p}$ into (7) amounts to $\frac{\vec{e}_{\odot, \oplus} \cdot \vec{p}}{\vec{e}_{\odot, \oplus \cdot} \cdot \vec{n}_{\odot, \oplus}} \vec{n}_{\odot, \oplus}=\frac{w_{\oplus}-w_{\odot}}{d_{\odot, \oplus} \vec{\odot}_{\odot, \oplus} \cdot \vec{\oplus}_{\odot, \oplus}} \vec{n}_{\odot, \oplus}=$ $\frac{w_{\oplus}-w_{\odot}}{\overline{\bar{x}_{\odot} x_{\oplus} \cdot \vec{n}_{\odot}, \oplus}} \vec{n}_{\odot, \oplus}$. Combining the obtained identities, we get (6) from (7).

2.3. Cartesian DDFV meshes in 3D. Let us give one very simple yet important concrete example of a 3D DDFV mesh as described above. It is suitable for parallelepiped domains and their unions.

For the sake of simplicity, we take the unit cube $\Omega=[0,1]^{3}$ and partition it into $N^{3}$ primal cubic volumes of edge $\frac{1}{N}$ (neither the cubic form, nor the uniformity of the meshes is important; the construction generalizes to non-uniform cartesian meshes). Then one easily sees that diamonds are octahedrons built on two primal cubes' centers $x_{K_{\odot}}, x_{K_{\oplus}}$ and on the square interface $K_{\odot} \mid K_{\oplus}$ between them. If one chooses for $x_{K_{\odot} \mid K_{\oplus}}$ the center of symmetry of $K_{\odot} \mid K_{\oplus}$, then the interior dual volumes are also cubes of the same edge $\frac{1}{N}$ centered at the vertices of the primal mesh that do not lie on $\partial \Omega$. The boundary dual volumes are either $1 / 8$ th of the standard cube (at the corners of $\Omega$ ) or the quarter-cubes (at the edges of $\Omega$, excepting the corners) or the half-cubes (on the faces of $\Omega$, excepting the edges). 


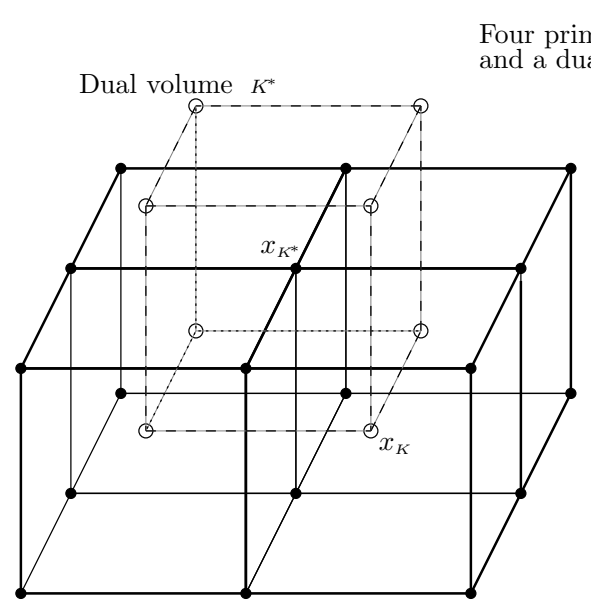

Primal volume $K$



Primal volume $K_{\odot}$

Figure 3. Cartesian DDFV mesh in 3D and an associated diamond

To give the entries of the associated discrete gradient, for the sake of being definite we consider a diamond $D=D^{K_{\odot} \mid K_{\oplus}}$ with the interface $K_{\odot} \mid K_{\oplus}$ parallel to the horizontal plane $x O y$ and with $\overrightarrow{x_{\odot} x_{\oplus}}$ pointing up in the direction $O z$ (see Figure 3). We take the convention that the $O x$ axis points from West to East and the $O y$ axis points from South to North. Then we mark the vertices of the square $K \mid L$ counterclockwise (with respect to the orientation given by $O z$ and $\overrightarrow{x_{\odot} x_{\oplus}}$ ) by $x_{K_{\mathrm{SW}}^{*}}, x_{K_{\mathrm{SE}}^{*}}, x_{K_{\mathrm{NE}}^{*}}$ and $x_{K_{\mathrm{NW}}^{*}}$, with an obvious "cartographic" interpretation of the notation.

With this notation, applying the reconstruction formula (5) one finds that the entry $\nabla_{D} w^{\mathfrak{T}}$ of $\nabla^{\mathfrak{T}} w^{\mathfrak{T}}$ is computed as

$$
\begin{aligned}
\nabla_{D} w^{\mathfrak{T}}= & \frac{w_{K_{\oplus}}-w_{K_{\odot}}}{1 / N} \vec{k} \\
& +\frac{1}{2}\left(\frac{w_{K_{\mathrm{NE}}^{*}}-w_{K_{\mathrm{NW}}^{*}}}{1 / N}+\frac{w_{K_{\mathrm{SE}}^{*}}-w_{K_{\mathrm{SW}}^{*}}}{1 / N}\right) \vec{i}+\frac{1}{2}\left(\frac{w_{K_{\mathrm{NE}}^{*}}-w_{K_{\mathrm{SE}}^{*}}}{1 / N}+\frac{w_{K_{\mathrm{NW}}^{*}}-w_{K_{\mathrm{SW}}^{*}}}{1 / N}\right) \vec{j}
\end{aligned}
$$

(recall that $\left.d_{K_{\odot}, K_{\oplus}}=d_{K_{\mathrm{NE}}^{*}, K_{\mathrm{NW}}^{*}}=\ldots=\frac{1}{N}\right)$; here the triple $(\vec{i}, \vec{j}, \vec{k})$ is the canonical basis of $\mathbb{R}^{3}$.

Further, let $K$ be an interior primal volume. For the six diamonds that intersect $K$, we introduce the specific notation $D_{\mathrm{abv}}(K), D_{\mathrm{blw}}(K), D_{\mathrm{E}}(K), D_{\mathrm{W}}(K)$ and $D_{\mathrm{N}}(K), D_{\mathrm{S}}(K)$. For instance, $D_{\mathrm{S}}(K)$ contains the south-ward face of $K$ (the exterior unit normal vector to $\partial K \cap D_{\mathrm{S}}(K)$ is the vector $-\vec{j}$ ), and $D_{\mathrm{abv}}(K)$ contains the upper face of $K$ (the corresponding exterior normal vector is $\vec{k}$ ). With this notation, applying formula (3) one finds that the entry $\operatorname{div}_{K} \overrightarrow{\mathcal{F}}^{\mathfrak{I}}$ of $\operatorname{div}^{\mathfrak{T}} \overrightarrow{\mathcal{F}}^{\mathfrak{z}}$ is computed as

$\operatorname{div}_{K} \overrightarrow{\mathcal{F}}^{\mathfrak{I}}=\frac{1}{1 / N^{3}}\left\{\frac{1}{N^{2}}\left(\overrightarrow{\mathcal{F}}_{D_{\mathrm{E}}(K)}-\overrightarrow{\mathcal{F}}_{D \mathrm{~W}}(K)\right) \cdot \vec{i}+\frac{1}{N^{2}}\left(\overrightarrow{\mathcal{F}}_{D_{\mathrm{N}}(K)}-\overrightarrow{\mathcal{F}}_{D \mathrm{~S}(K)}\right) \cdot \vec{j}+\frac{1}{N^{2}}\left(\overrightarrow{\mathcal{F}}_{D_{\mathrm{abv}}(K)}-\overrightarrow{\mathcal{F}}_{D_{\mathrm{blw}}(K)}\right) \cdot \vec{k}\right\}$.

The formula for the entries of $\operatorname{div}_{K^{*}} \overrightarrow{\mathcal{F}}^{\mathfrak{T}}$ has an entirely similar form.

The above meshes and discrete operators can be compared to the $2 \mathrm{D}$ constructions of $[7,8]$ and to the $2 \mathrm{D}$ and $3 \mathrm{D}$ "complementary volumes" constructions of [39, 40, 55]. As the schemes of [8] and of [39], the above DDFV scheme tends to avoid preferred orientations (cf. the discussion in [39]).

\section{THE DISCRETE DUALITY PROPERTY}

Let us define the convenient multiplication of discrete functions/discrete fields and state our main result.

- Recall that $\mathbb{R}^{\mathfrak{T}}$ is the space of all discrete functions on $\Omega$. For $w^{\mathfrak{s}}, v^{\mathfrak{I}} \in \mathbb{R}^{\mathfrak{T}}$, set

$$
\llbracket w^{\mathfrak{T}}, v^{\mathfrak{T}} \rrbracket_{\Omega}=\frac{1}{3} \sum_{K \in \mathfrak{M}^{\circ}} \operatorname{Vol}(K) w_{K} v_{K}+\frac{2}{3} \sum_{K^{*} \in \mathfrak{M}^{*}} \operatorname{Vol}\left(K^{*}\right) w_{K^{*}} v_{K^{*}}
$$


it is clear that $\llbracket \cdot, \cdot \rrbracket_{\Omega}$ is a scalar product on $\mathbb{R}^{\mathfrak{T}}$.

Remark 3.1. Notice that, contrarily to the 2D DDFV method, the role of the primal and the dual meshes in 3D CeVe-DDFV methods is not symmetric; this asymmetry is also reflected by Remark 2.2. In $\mathbb{R}^{d}$, the primal mesh would account for $\frac{1}{d}$ of the product, and the dual mesh, for $\frac{d-1}{d}$ (see [5]).

Note that in the 3D CeVe-DDFV scheme developed by Pierre et al. (see [57, 24, 25, 23]), the weights in the scalar product (9) are both equal to $\frac{1}{3}$; but, because the dual mesh in $[57,24,25,23]$ covers twice the domain $\Omega \subset \mathbb{R}^{3}$, its "weight" is doubled with respect with the "weight" of the primal mesh. This is similar to what happens in our formula (9). As to the 3D CeVeFE-DDFV scheme developed by Coudière and Hubert (see [19, 20, 21]), a "triple" mesh is involved, and the associated scalar product takes the contributions of each of the meshes with the equal weights $\frac{1}{3}$.

- Recall that $\left(\mathbb{R}^{3}\right)^{\mathcal{D}}$ is the space of all discrete fields on $\Omega$. For $\overrightarrow{\mathcal{F}}^{\mathfrak{T}}, \overrightarrow{\mathcal{G}}^{\mathfrak{T}} \in\left(\mathbb{R}^{3}\right)^{\mathcal{D}}$, set

$$
\left\{\left\{\overrightarrow{\mathcal{F}}^{\mathfrak{T}}, \overrightarrow{\mathcal{G}}^{\mathfrak{T}}\right\}_{\Omega}=\sum_{D \in \mathfrak{D}} \operatorname{Vol}(D) \overrightarrow{\mathcal{F}}_{D} \cdot \overrightarrow{\mathcal{G}}_{D}\right.
$$

it is clear that $\left\{\{, \cdot\}_{\Omega}\right.$ is a scalar product on $\left(\mathbb{R}^{3}\right)^{\mathcal{D}}$.

Proposition 3.2. The discrete divergence and gradient operators - $\operatorname{div}^{\mathfrak{T}}, \nabla^{\mathfrak{T}}$ defined in Section 2 are linked by the following duality property :

$$
\forall w^{\overline{\mathfrak{T}}} \in \mathbb{R}_{0}^{\overline{\mathfrak{T}}} \quad \forall \overrightarrow{\mathcal{F}}^{\mathfrak{T}} \in\left(\mathbb{R}^{3}\right)^{\mathfrak{D}} \quad \llbracket-\operatorname{div}^{\mathfrak{T}} \overrightarrow{\mathcal{F}}^{\mathfrak{T}}, w^{\mathfrak{T}} \rrbracket_{\Omega}=\left\{\left\{\overrightarrow{\mathcal{F}}^{\mathfrak{T}}, \nabla^{\mathfrak{T}} w^{\overline{\mathfrak{T}}}\right\}\right\}_{\Omega} .
$$

Before turning to the proof, let us introduce the partition of $\Omega$ into subdiamonds (cf. [5, 4]), as used in the proof, and give two more formulas for the discrete divergence operators (namely, (12) and (15) below).

If $K, L \in \overline{\mathfrak{M}^{0}}$ are neighbours, and $x_{K^{*}}, x_{L^{*}}$ are neighbour vertices of the corresponding interface $K L$, then the union of the four elements ${ }^{5} T_{K^{*} ; L^{*}}^{K ; L}, T_{L^{*}, K^{*}}^{K ; L}, T_{K^{*}, L^{*}}^{L ; K}$, and $T_{L^{*}, K^{*}}^{L ; K}$ is called subdiamond and denoted by $S_{K^{*} L^{*}}^{K \mid L}$. In this way, each diamond $D^{K \mid L}$ gives rise to $l$ subdiamonds ( $l$ being the number of vertices of $K L$ ).

We denote by $\mathfrak{S}$ the set of all subdiamonds. Generic suddiamond in $\mathfrak{S}$ is denoted by $S$. Each subdiamond is associated with a unique interface $K L$, and thus with a unique diamond $D^{K \mid L}$. We will write $S \sim D$ to signify that $S$ is associated with $D$; in this case, we set $\overrightarrow{\mathcal{F}}_{\mathrm{S}}=\overrightarrow{\mathcal{F}}_{D}$ for a discrete field $\overrightarrow{\mathcal{F}}$. Further, if $x_{K}$ (resp., $x_{K^{*}}$ ) is a vertice of $S$, then we write $S \sim K$ (resp., $S \sim K^{*}$ ).

Lemma 3.3. Formulas (3) can be rewritten under the form

$$
\begin{aligned}
\operatorname{div}_{K} \overrightarrow{\mathcal{F}}^{\mathfrak{T}} & =\frac{1}{2 \operatorname{Vol}(K)} \sum_{S \sim K}(-1)^{\epsilon_{S}^{K}}\left\langle\overrightarrow{\mathcal{F}}_{S}, \overrightarrow{x_{\odot, \oplus}^{*} x_{i, i+1}^{*}}, \overrightarrow{x_{i}^{*} x_{i+1}^{*}}\right\rangle, \\
\operatorname{div}_{K^{*}} \overrightarrow{\mathcal{F}}^{\mathfrak{T}} & =\frac{1}{2 \operatorname{Vol}\left(K^{*}\right)} \sum_{S \sim K^{*}}(-1)^{\epsilon_{S}^{K^{*}}}\left\langle\overrightarrow{\mathcal{F}_{S}}, \overrightarrow{x_{\odot} x_{\oplus}}, \overrightarrow{x_{\odot, \oplus}^{*} x_{i, i+1}^{*}}\right\rangle .
\end{aligned}
$$

In formulas (12), we mean that each subdiamond $S$ associated with $K$ (resp., with $K^{*}$ ) has the form $S=S_{K_{i}^{*} \mid K_{i+1}^{*}}^{K_{\oplus} \mid K_{\oplus}}$, with some $K_{\odot}, K_{\oplus}, K_{i}^{*}, K_{i+1}^{*}$; the notation under the sign " $\sum$ " refers to $S=S_{K_{i}^{*} \mid K_{i+1}^{*}}^{K_{\odot} \mid K_{\oplus}}$ (see Figure 2). Because $K$ may coincide either with $K_{\odot}$ or with $K_{\oplus}$ (similarly, $K^{*}$ may be $K_{i}^{*}$ or $\left.K_{i+1}^{*}\right)$, the "sign selectors"

$$
\epsilon_{\mathrm{S}}^{K}:=\left\{\begin{array}{l}
0, \text { if } K=K_{\odot} \\
1, \text { if } K=K_{\oplus}
\end{array} \quad, \quad \epsilon_{\mathrm{S}}^{K^{*}}:=\left\{\begin{array}{l}
0, \text { if } K^{*}=K_{i}^{*} \\
1, \text { if } K^{*}=K_{i+1}^{*}
\end{array}\right.\right.
$$

are introduced in (12).

Proof of Lemma 3.3 : Fix a subdiamond $S \in \mathfrak{S}, S=S_{K_{i}^{*} \mid K_{i+1}^{*}}^{K_{\odot} \mid K_{\oplus}}$. The subdiamond contributes twice to each of the formulas (3) corresponding to $K=K_{\odot}$, to $K=K_{\oplus}$, to $K^{*}=K_{i}^{*}$, and to

\footnotetext{
${ }^{5}$ This definition should be generalized if the constraint $x_{K} \in K$ is dropped; see Remark 4.1.
} 



FIGURE 4. Element. Subdiamond: $\sigma_{\mathrm{S}}, \sigma_{\mathrm{S}_{\oplus}}, \sigma_{\mathrm{S} \odot}$ and their normal vectors.

$K^{*}=K_{i+1}^{*}$; each contribution is brought by two of the four elements $T \in \mathcal{T}$ that constitute $S$. We denote the four elements by

$$
T_{i}^{\odot}:=T_{K_{i}^{*} ; K_{i+1}^{*}}^{K_{\odot} ; K_{\oplus}}, \quad T_{i+1}^{\odot}:=T_{K_{i+1}^{*} ; K_{i+1}^{*}}^{K_{\odot} ; K_{\oplus}} \quad \text { and } \quad T_{i}^{\oplus}:=T_{K_{i}^{*} ; K_{i+1}^{*}}^{K_{\oplus} ; K_{\odot}}, \quad T_{i+1}^{\oplus}:=T_{K_{i+1}^{*}, K_{i+1}^{*}}^{K_{\oplus}^{*} ; K_{\odot}} .
$$

It is convenient to split $S=S_{K_{i}^{*} \mid K_{i+1}^{\oplus}}^{K_{\odot} \mid K_{\oplus}}$ into the "lower" and the "upper" parts

$$
S_{\odot}:=T_{i}^{\odot} \cup T_{i+1}^{\odot} \quad \text { and } \quad S_{\oplus}:=T_{i}^{\oplus} \cup T_{i+1}^{\oplus}
$$

(see Figure 4). Each of the two parts contains one flat portion of the interface $K_{i}^{*} \mid K_{i+1}^{*}$, and they are separated by the common face $K_{\odot} \mid K_{\oplus} \cap S$. We denote these faces as follows:

$$
\sigma_{\mathrm{S}}:=K_{\odot}\left|K_{\oplus} \cap S, \quad \sigma_{\odot}^{*}:=K_{i}^{*}\right| K_{i+1}^{*} \cap S \odot, \quad \sigma_{\mathrm{S} \oplus}^{*}:=K_{i}^{*} \mid K_{i+1}^{*} \cap S_{\oplus} .
$$

The areas of $\sigma_{\mathrm{S}}, \sigma_{\mathrm{S}_{\odot}}^{*}, \sigma_{\mathrm{S} \oplus}^{*}$ are denoted by $m_{\mathrm{S}}, m_{\mathrm{S}_{\odot}}^{*}, m_{\mathrm{S} \oplus}^{*}$, respectively; one calculates them from the areas of faces of the elements $T_{i}^{\odot}, T_{i}^{\oplus}, T_{i+1}^{\odot}, T_{i+1}^{\oplus}$.

We denote by $\vec{n}_{\mathrm{S}}, \vec{n}_{\mathrm{S}_{\odot}}^{*}$, $\vec{n}_{\mathrm{S}_{\oplus}}^{*}$ the unit normal vectors to $\sigma_{\mathrm{S}}, \sigma_{\mathrm{S}_{\odot}}^{*}$, $\sigma_{\mathrm{S}_{\oplus}}^{*}$, respectively, with the orientations that are chosen so that to satisfy

$$
\vec{n}_{\mathrm{S}}=\vec{n}_{\odot, \oplus} \quad \text { and }\left\langle\vec{n}_{\varsigma_{\odot}}^{*}, \vec{n}_{\odot, \oplus}, \overrightarrow{x_{\odot, \oplus}^{*} x_{i, i+1}^{*}}\right\rangle \geq 0, \quad\left\langle\vec{n}_{\varsigma_{\oplus}}^{*}, \vec{n}_{\odot, \oplus}, \overrightarrow{x_{\odot, \oplus}^{*} x_{i, i+1}^{*}}\right\rangle \geq 0 .
$$

Thanks to the constraints $x_{\odot} \in K_{\odot}, x_{\oplus} \in K_{\oplus}$ and to the orientation choice $\vec{n}_{\mathrm{S}} \cdot \overrightarrow{x_{\odot} x_{\oplus}}=d_{\odot, \oplus} \vec{n}_{\odot, \oplus}$. $\vec{e}_{\odot, \oplus}>0$, vector $\vec{n}_{\mathrm{S}}$ points from $K_{\odot}$ to $K_{\oplus}$; thus by definition (13) of $\epsilon_{S}^{K}$, the vector $(-1)^{\epsilon_{S}^{K}} \vec{n}_{\mathrm{S}}$ is the unit normal vector to $\sigma_{\mathrm{S}} \subset \partial K$ exterior to $K$, in the case $K=K_{\odot}$ and also in the case $K=K_{\oplus}$. Thus for $K=K_{\odot}$ and for $K=K_{\oplus}$, for all element $T \subset S$ such that $T$ is associated to $K$, we have $\vec{n}_{T}=(-1)^{\epsilon_{S}^{K}} \vec{n}_{\mathrm{S}}$.

Similarly, having chosen $x_{\odot, \oplus}^{*} \in K_{\odot} \mid K_{\oplus}$ we ensured that $\vec{n}_{\varsigma_{\odot}}^{*}, \vec{n}_{\mathrm{S} \oplus}^{*}$ point from $K_{i}^{*}$ to $K_{i+1}^{*}$, thanks to (14) and to the fact that the vertices of $K_{\odot} \mid K_{\oplus}$ are numbered according to the orientation of $\vec{n}_{\odot, \oplus}$. By (13), (-1) $\epsilon_{S}^{K^{*}} \vec{n}_{\mathrm{S}_{\odot}}^{*},(-1)^{\epsilon_{S}^{K^{*}}} \vec{n}_{\mathrm{S} \oplus}^{*}$ are the unit normal vectors to the flat portions $\sigma_{\mathrm{S}_{\odot}}^{*}$, $\sigma_{\mathrm{S} \oplus}^{*}$ of $\partial K^{*}$, exterior to $K^{*}$. Thus for $K^{*}=K_{i}^{*}$ and for $K^{*}=K_{i+1}^{*}$, for all element $T \subset S_{\odot}$ (resp., $\left.T \subset S_{\oplus}\right)$ such that $T \sim K^{*}$, we have $\vec{n}_{T}^{*}=(-1)^{\epsilon_{S}^{K^{*}}} \vec{n}_{\triangleright \odot}^{*} \quad\left(\right.$ resp., $\vec{n}_{T}^{*}=\left(-1 \epsilon^{\epsilon_{S}^{K^{*}}} \vec{n}_{\mathrm{S} \oplus}^{*}\right)$.

Now we see that (3) rewrites under the form

$$
\begin{aligned}
\operatorname{div}_{K} \overrightarrow{\mathcal{F}}^{\mathfrak{T}} & =\frac{1}{\operatorname{Vol}(K)} \sum_{\mathrm{S} \sim K} m_{\mathrm{S}} \overrightarrow{\mathcal{F}}_{\mathrm{S}} \cdot(-1)^{\epsilon_{S}^{K}} \vec{n}_{\mathrm{S}}, \\
\operatorname{div}_{K^{*}} \overrightarrow{\mathcal{F}}^{\mathfrak{T}} & =\frac{1}{\operatorname{Vol}\left(K^{*}\right)} \sum_{\mathrm{S} \sim K^{*}} \overrightarrow{\mathcal{F}}_{\mathrm{S}} \cdot(-1)^{\epsilon_{S}^{K^{*}}}\left(m_{\mathrm{S} \odot}^{*} \vec{n}_{\mathrm{S} \odot}^{*}+m_{\mathrm{S} \oplus}^{*} \vec{n}_{\mathrm{S} \oplus}^{*}\right) .
\end{aligned}
$$


Finally, notice that the above definitions and the standard properties of the inner product " $\times$ " of $\mathbb{R}^{3}$ yield

$$
\begin{aligned}
& m_{\mathrm{S}} \vec{n}_{\mathrm{S}}=\frac{1}{2} \overrightarrow{x_{\odot, \oplus}^{*} x_{i, i+1}^{*}} \times \overrightarrow{x_{i}^{*} x_{i+1}^{*}}, \\
& m_{\mathrm{S} \odot}^{*} \vec{n}_{\mathrm{S} \odot}^{*}+m_{\mathrm{S} \oplus}^{*} \vec{n}_{\mathrm{S} \oplus}^{*}=\frac{1}{2} \overrightarrow{x_{\odot} x_{\odot, \oplus}^{*}} \times \overrightarrow{x_{\odot, \oplus}^{*} x_{i, i+1}^{*}}+\frac{1}{2} \overrightarrow{x_{\odot, \oplus}^{*} x_{\oplus}} \times \overrightarrow{x_{\odot, \oplus}^{*} x_{i, i+1}^{*}}=\frac{1}{2} \overrightarrow{x_{\odot} x_{\oplus}} \times \overrightarrow{x_{\odot, \oplus}^{*} x_{i, i+1}^{*}} .
\end{aligned}
$$

Using equalities (16), from (15) we deduce (12).

Proof of Proposition 3.2 : The proof is by direct calculation, using the summation-by-parts procedure.

Denote the product $\llbracket-\operatorname{div}^{\mathfrak{T}} \overrightarrow{\mathcal{F}} \mathfrak{\mathfrak { I }}, w^{\mathfrak{s}} \|_{\Omega}$ by $Z$. First, we put together the terms in $Z$ corresponding to adjacent couples of primal and dual volumes. This amounts to make the summation over all subdiamonds $S=S_{K_{i}^{*} \mid K_{i+1}^{\oplus}}^{K_{\oplus} \mid K_{i}}$ (see Figures 2, 4 for the notation in $S$ ); ghost terms corresponding to the boundary volumes can be added, because $w^{\bar{\Sigma}}$ is zero on the boundary volumes. With the expression (9) of $\llbracket \cdot, \cdot \rrbracket_{\Omega}$, using formulas (12) of Lemma 3.3, taking into account the signs selectors' convention (13) while summing by parts, we find

$$
\begin{aligned}
Z & =\sum_{S \in \mathfrak{S}, S=S_{K_{i}^{*} \mid K_{\oplus+1}^{*}}^{K_{\odot}}} \overrightarrow{\mathcal{F}}_{\mathrm{S}} \cdot\left(\frac{1}{3}\left(w_{\oplus}-w_{\odot}\right)\left[\overrightarrow{x_{\odot, \oplus}^{*} x_{i, i+1}^{*}} \times \overrightarrow{x_{i}^{*} x_{i+1}^{*}}\right]+\frac{1}{3}\left(w_{i+1}^{*}-w_{i}^{*}\right)\left[\overrightarrow{x_{\odot} x_{\oplus}} \times \overrightarrow{x_{\odot, \oplus}^{*} x_{i, i+1}^{*}}\right]\right) \\
& =\sum_{S \in \mathfrak{S}, S=S_{K_{i}^{*} \mid K_{\oplus+1}^{*}}^{K_{\odot}}} \overrightarrow{\mathcal{F}}_{\mathrm{S}} \cdot\left(\operatorname{Vol}\left(S_{K_{i}^{*} \mid K_{i+1}^{*}}^{K_{\odot}}\right) \frac{w_{\oplus}-w_{\odot}}{\overline{x_{\odot} x_{\oplus} \cdot \vec{n}_{\odot, \oplus}}} \vec{n}_{\odot, \oplus}+\frac{1}{3}\left(w_{i+1}^{*}-w_{i}^{*}\right)\left[\overrightarrow{x_{\odot}} \overrightarrow{x_{\oplus}} \times \overrightarrow{x_{\odot, \oplus}^{*} x_{i, i+1}^{*}}\right]\right) .
\end{aligned}
$$

Next, we put together the terms corresponding to the subdiamonds $S$ associated with the same diamond $D$; since $\overrightarrow{\mathcal{F}}_{\mathrm{S}}=\overrightarrow{\mathcal{F}}_{D}$ in this case, this amounts to make the summation over all diamonds $D=D^{K_{\odot} \mid K_{\oplus}}$ (see Figures 2, 6 for the notation in $D$ ). We get $Z=\sum_{D \in \mathfrak{\mathcal { D }}} \overrightarrow{\mathcal{F}}_{D} \cdot \vec{Z}_{D}$ with

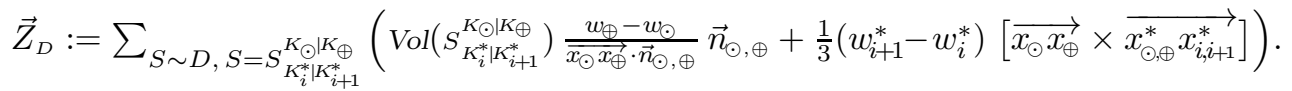

Thanks to Lemma 2.4, the above expression $\vec{Z}_{D}$ equals $\operatorname{Vol}(D) \nabla_{D}^{\mathfrak{\tau}} w^{\overline{\mathfrak{s}}}$. According to the ex-

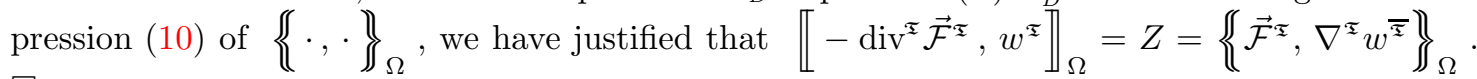

\section{ON THE CHOICE OF THE FACE CENTERS $x_{K \mid L}$ AND OTHER GENERALiZATIONS}

Here we provide a series of remarks that discuss and generalize the above construction.

\section{Remark 4.1. (an overview of the different constraints)}

Given a partition of $\Omega$ into convex disjoint open subsets $K$, one can always construct a "double" mesh $\mathfrak{T}$ satisfying the constraints on the choice of $x_{K}, x_{K \mid L}, x_{K^{*} \mid L^{*}}$ imposed in Section 2. But if one wants to use some simpler constructions such as the classical Voronoï dual mesh in Example 4.3 below, some of the below generalizations are needed.

- The barycenter (middlepoint) choice $x_{K^{*} \mid L^{*}}=\frac{1}{2}\left(x_{K^{*}}+x_{L^{*}}\right)$ on the edges cannot be relaxed.

This choice is in the heart of the consistency property of Proposition 2.3 (see the proof of Lemma 5.1).

- The constraint $x_{K \mid L} \in K \mid L$ can be relaxed, and this generalization is important.

In particular, it may be convenient (as e.g. in Example 4.3 below) to let $x_{K \mid L}$ be the point of intersection of the plane containing the interface $K \mid L$ with the line passing through the centers $x_{K}, x_{L}$. Even if $x_{K} \in K$ and the volumes are convex, the intersection point can fall outside $K \mid L$.

In fact, the property $x_{K \mid L} \in K \mid L$ is only needed to ensure that the subdiamond volume, $\operatorname{Vol}(S)=\frac{1}{6}\left\langle\overrightarrow{x_{\odot} x_{\oplus}}, \overrightarrow{x_{\odot, \oplus}^{*} x_{i, i+1}^{*}}, \overrightarrow{x_{i}^{*} x_{i+1}^{*}}\right\rangle$, is positive. Yet, this positivity restriction does not appear in Lemma 5.1, Corollary 5.2 and Proposition 2.3. Let us stress that all the formulas in terms of vector and mixed products given in this note can be used without changes, if 
$x_{K L L}$ belongs to the plane containing $K \mid L$ (see in particular Remark 5.3). But it becomes necessary to generalize the notions of $K^{*}$ and $\operatorname{Vol}\left(K^{*}\right)$, allowing for subdiamonds and elements with negative volume. We througly illustrate the situation in Remark 4.2 below.

- The requirement that $x_{K}$ belong to $K$ can be relaxed, which is important as well.

The definition of diamonds and subdiamonds becomes a bit more complicated in this case, because some elements $T \in \mathcal{T}$ may have negative volume; the situation in entirely similar to that of Remark 4.2 below. In order to have $\operatorname{Vol}(D)>0$, it suffices to guarantee that the normal to $\left.K_{\odot}\right|_{\oplus}$ vector $\vec{n}_{\odot, \oplus}$ (which, by definition, forms an acute angle with $\overrightarrow{x_{\odot} x_{\oplus}}$ ) point from $K_{\odot}$ to $K_{\oplus}$. To this end, the Delaunay property is required in Example 4.3.

- The convexity constraint on the primal volumes $K$ can be relaxed.

E.g., if each volume $K$ is star-shaped with respect to some point $x_{K}$, the construction goes on without any change. More generally, we can even admit non-planar faces, by separating them into planar parts.

\section{Remark 4.2. (on the constraint $x_{K \mid L} \in K \mid L$ and its relaxation)}

(i) Under the assumptions that $x_{K} \in K$ for all $K, x_{K \mid L} \in K \mid L$ for all $K \mid L$, the set of all elements $\mathcal{T}$ is a partition of $\Omega$, and each of the partitions $\mathfrak{M}^{o}, \overline{\mathfrak{M}^{*}}, \mathfrak{D}$ of $\Omega$ is obtained by combining elements. More exactly, we have $K=\bigcup T_{K^{*} ; L^{*}}^{K ; L}$, where the union runs over all $L \in \mathcal{N}(K)$ and all $K^{*}, L^{*}$ which are neighbour vertices of the polygon $K \mid L$. Similarly, $K^{*}=\bigcup T_{K^{*} ; L^{*}}^{K ; L}$, where the union runs over all $K, L^{*}$ such that $T_{K^{*} ; L^{*}}^{K ; L}$ makes sense. Finally,

$$
D^{K \mid L}=\bigcup\left(T_{K^{*} ; L^{*}}^{K ; L} \cup T_{L^{*} ; K^{*}}^{K ; L} \cup T_{K^{*} ; L^{*}}^{L ; K} \cup T_{L^{*} ; K^{*}}^{L ; K}\right)=\bigcup S_{K^{*} L^{*}}^{K L},
$$

where the union runs over all couples $\left\{K^{*}, L^{*}\right\}$ of neighbour vertices of the interface $K \mid L$.

When an element $T \in \mathcal{T}$ contributes to the construction of $K$, we say that $T$ is associated with $K$, and write $T \sim K$. We therefore have $K=\bigcup_{T \sim K} T$, and $\operatorname{Vol}(K)=\sum_{T \sim K} \operatorname{Vol}(T)$.

Analogous meaning is given to the notation $T \sim K^{*}, T \sim D$, and $S \sim D, T \sim S$; e.g.,

$$
D=\bigcup_{\mathrm{S} \sim D} S, \quad \text { and } \quad \operatorname{Vol}(D)=\sum_{\mathrm{S} \sim D} \operatorname{Vol}(S) .
$$

In each case, the relation " $\sim$ " simply means the inclusion " $\subset$ ".

(ii) Now, for one example where $x_{K \mid L} \notin K \mid L$, let $K \mid L$ be a triangle with vertices denoted by $x_{K^{*}}, x_{L^{*}}, x_{M^{*}}$, with obtuse angle at $x_{L^{*}}$; let $x_{K \mid L}$ be the center of circonscribed circle of the triangle which therefore falls outside $K \mid L$ (this situation occurs in Example 4.3). Instead of the decomposition

$$
D^{K \mid L}=\bigcup_{S \sim D} S=S_{K^{*} \mid L^{*}}^{K \mid L} \cup S_{L^{*} \mid M^{*}}^{K \mid L} \cup S_{M^{*} \mid K^{*}}^{K \mid L}
$$

we now have

$$
D^{K \mid L}=\left(S_{K^{*} \mid L^{*}}^{K \mid L} \cup S_{L^{*} \mid M^{*}}^{K \mid L}\right) \backslash S_{M^{*} \mid K^{*}}^{K \mid L}
$$

But if (with the notation of Figures 2, 4) we keep the formula

$$
\operatorname{Vol}(s)=\frac{1}{6}\left\langle\overrightarrow{x_{\odot} x_{\oplus}}, \overrightarrow{x_{\odot, \oplus}^{*} x_{i, i+1}^{*}}, \overrightarrow{x_{i}^{*} x_{i+1}^{*}}\right\rangle
$$

for the volume of $S$, we see that $\operatorname{Vol}\left(S_{M^{*} \mid K^{*}}^{K \mid L}\right)$ becomes negative, and cancellations lead to

$$
\operatorname{Vol}\left(D^{K \mid L}\right)=\left|\operatorname{Vol}\left(S_{K^{*} \mid L^{*}}^{K \mid L}\right)\right|+\left|\operatorname{Vol}\left(S_{L^{*} \mid M^{*}}^{K \mid L}\right)\right|-\left|\operatorname{Vol}\left(S_{M^{*} \mid K^{*}}^{K K L}\right)\right|=\operatorname{Vol}\left(S_{K^{*} \mid L^{*}}^{K \mid L}\right)+\operatorname{Vol}\left(S_{L^{*} \mid M^{*}}^{K \mid L}\right)+\operatorname{Vol}\left(S_{M^{*} \mid K^{*}}^{K \mid L}\right)=\sum_{S \sim D} \operatorname{Vol}(S) .
$$

We see that the set-theoretic relation in (17) looses its sense, but the formula for $\operatorname{Vol}(D)$ keeps working. Similarly, a primal volume $K$ is a set of points of $\Omega$ that can be obtained by the operations " $\cup$ ", " \" from the elements $T$ associated with $K$, and $\operatorname{Vol}(K)=\sum_{\mathrm{S} \sim K} \operatorname{Vol}(T)$; the (signed) volume of $T$ can be computed by a formula similar to (18). Let us point out that $\operatorname{sign}(\operatorname{Vol}(T))=\operatorname{sign}(\operatorname{Vol}(S))$ when $T \subset S$, and $\operatorname{Vol}(S)=\sum_{T \subset S} \operatorname{Vol}(T)$. 
The general situation is the same as in the above example. Notice that neither $\mathcal{T}$, nor $\mathfrak{S}$ form a partition of $\Omega$; but each one forms a "signed partition" of $\Omega$ in the sense that

$$
\sum_{T \in \mathcal{T}} \operatorname{sign}(\operatorname{Vol}(T)) \mathbb{1}_{T}(x)=1 \quad \text { and } \quad \sum_{\mathrm{S} \in \mathfrak{S}} \operatorname{sign}(\operatorname{Vol}(S)) \mathbb{1}_{\mathrm{S}}(x)=1 \quad \text { a.e. on } \Omega
$$

(here $\mathbb{1}_{A}(\cdot)$ stands for the characteristic function of a set $A \subset \Omega$ ).

The situation with dual volumes $K^{*}$ can be more intricated: $K^{*}$ may in general consist of a "positive" and a "negative" part, to which no set-theoretical meaning can be given ${ }^{6}$; but we can give the sense of $\sum_{T \sim K^{*}} \operatorname{Vol}(T)$ to $\operatorname{Vol}\left(K^{*}\right)$. In this case, let us call $K^{*}$ a generalized dual volume. Here a constraint appears on the choice of the family $\left(x_{K \mid L}\right)_{K \mid L}$ of the face centers: one should keep $\operatorname{Vol}\left(K^{*}\right)>0$, in order that $(9)$ be a scalar product.

Similar interpretation can be given to the discrete divergence formulas (12). In (12), the normal flux of $\overrightarrow{\mathcal{F}}_{\mathrm{S}}$ through $\sigma_{S}$ is automatically taken with the same sign as $\operatorname{sign}(\operatorname{Vol}(S))$, and the cancellations in the expression

$$
\sum_{\mathrm{S} \in \mathcal{N}(K)} m_{\mathrm{S}} \overrightarrow{\mathcal{F}}_{\mathrm{S}} \cdot(-1)^{\epsilon_{S}^{K}} \vec{n}_{\mathrm{S}}
$$

make it be equal to the normal flux of the field $\overrightarrow{\mathcal{F}}$ through $\partial_{K}$. Notice that the relation " $S \sim K$ " should be understood in the sense that $S \supset T$ for some $T \sim K$. Similarly, for a (possibly generalized) control volume $K^{*}$, the flux through its (possibly generalized) boundary $\partial K^{*}$ is the sum of the signed contributions of the normal fluxes through $\sigma_{\mathrm{S} \odot}^{*}, \sigma_{\mathrm{S} \oplus}^{*} \subset S$ with $S \sim K^{*}$. Formulas (12) take care of this convention.

(iii) Clearly, these conventions should affect the discretization of source terms on the mesh $\boldsymbol{T}$. In the case (i) above, one naturally defines the projection of $f \in L^{1}(\Omega)$ on the space $\mathbb{R}^{\mathfrak{T}}$ of discrete functions by $\bar{f}^{\mathfrak{I}}=\left(\left(\bar{f}_{K}\right)_{K \in \mathfrak{M}^{o}},\left(\bar{f}_{K^{*}}\right)_{K^{*} \in \mathfrak{M}^{*}}\right)$ with $\bar{f}_{K}=\frac{1}{\operatorname{Vol}(K)} \int_{K} f, \bar{f}_{K^{*}}=\frac{1}{\operatorname{Vol}\left(K^{*}\right)} \int_{K^{*}} f$. But in the case (ii), we should rather generalize these formulas and write

$$
\bar{f}_{K^{*}}=\frac{1}{\operatorname{Vol}\left(K^{*}\right)} \sum_{T \sim K^{*}} \operatorname{sign}(\operatorname{Vol}(T)) \int_{T} f=\left(\sum_{T \sim K^{*}} \operatorname{Vol}(T)\right)^{-1} \sum_{T \sim K^{*}} \operatorname{sign}(\operatorname{Vol}(T)) \int_{T} f
$$

when $K^{*}$ is a generalized volume. The "signed partition" property (19) is a clue to the consistency of such projection operator. The "discrete functional analysis" properties given in [3] can be proved also in this generalized framework, under some additional restrictions such as $\operatorname{Vol}\left(K^{*}\right)>0$, $\operatorname{Vol}(D)>0$.

\section{Example 4.3. (Delaunay-Voronoï meshes)}

Let the primal mesh $\mathfrak{M}^{\circ}$ of $\Omega \subset \mathbb{R}^{3}$ be such that each $K \in \mathfrak{M}^{o}$ is a polyhedre admitting a circumscribed ball with center $x_{K}$ (for instance, a tetrahedron), and assume that all neighbour volumes $K, L$ satisfy the standard Delaunay condition. It follows that each face $K \mid L$ is an inscriptable polygon. Take for the dual mesh $\mathfrak{M}^{*}$, the standard Voronoï mesh constructed from the vertices of the primal mesh. This definition of $\mathfrak{M}^{*}$ enters our framework, with the following choice:

- the center $x_{K \mid L}$ of a face $K \mid L$ is the center of its circumscribed circle;

- the center $x_{K^{*} L^{*}}$ of an edge $K^{*} L^{*}$ is its middlepoint.

This construction does not guarantee that $x_{K \mid L} \in K \mid L$ nor that $x_{K} \in K$; thus we make appeal to the generalizations of the above Remarks 4.1, 4.2.

This "Delaunay-Voronoi" double scheme possesses the orthogonality property required for the approximation of entropy or renormalized solutions of (may be, degenerate) diffusion PDEs. See $[46,47,5]$, for examples of the use of this scheme.

Remark 4.4. Note that in order to get the mesh of Example 4.3, one can also reverse the construction procedure. Starting from a given set of points $x_{K^{*}}$, one constructs the Voronoï mesh which will play the role of the dual mesh $\mathfrak{M}^{*}$. A tetrahedrical primal mesh $\mathfrak{M}^{\circ}$ is obtained by joining apropriately the vertices of $\mathfrak{M}^{*}$; a slightly different convention on boundary volumes is needed in this case.

\footnotetext{
${ }^{6}$ We guess that this problem does not occur in Example 4.3, thanks to the Delaunay condition: the "negative" part of $K^{*}$ is completely cancelled by its "positive" part, as in the case of the primal volumes.
} 
Remark 4.5. Note that the discrete duality property of Proposition 3.2 is suitable for discrete functions satisfying the homogeneous Dirichlet condition on $\partial \Omega$. For different boundary conditions, see e.g. $[9,15,6]$. The case of the homogeneous Neumann boundary condition is briefly discussed in [3].

Remark 4.6. In $2 D$, the scheme described in [4] is much simpler than the DDFV scheme and still possesses the discrete duality property; the discrete duality for this scheme follows directly from the reconstruction property of the Appendix below.

This simpler scheme is well known for the case one starts with a triangulation of $\Omega \subset \mathbb{R}^{2}$ (this is essentially the "complementary volumes scheme", cf. [2, 40, 55, 39, 10, 59]). Elements of the triangulation play the role of diamonds of the DDFV scheme; in particular, discrete gradient is reconstructed as being constant per triangle. The dual Voronoï mesh of the triangulation is the finite volume mesh (these are the "complementary finite volumes", in the terminology of the papers $[40,55,39,59])$ on which one considers constant per volume discrete functions.

In the litterature, generalizations of such schemes to $3 \mathrm{D}$ were considered (see in particular [39]). To the authors' knowledge, the discrete duality property for the 3D "complementary finite volume" scheme only holds for very particular mesh geometries (e.g., uniform tetrahedral or rectangular meshes); see the discussion of [5, Appendix B].

\section{Numerical EXPERIMENTS FOR LINEAR DIFFUSION PROBLEMS}

For $\Omega \in \mathbb{R}^{3}$, consider the following linear diffusion problem:

$$
-\operatorname{div} A(x) \nabla u=f,\left.\quad u\right|_{\partial \Omega}=\bar{u},
$$

where $f$ is an $L^{2}$ source term, $(A(x))_{x \in \Omega}$ is a measurable (piecewise smooth, in many applications) family of uniformly bounded and coercive $3 \times 3$ matrices and $\bar{u}$ is a sufficiently regular boundary condition (when $\bar{u} \not \equiv 0$, the boundary conditions are taken into account at the centers of boundary volumes, cf. [9]).

The analogous $2 D$ problem was the example on which many $2 D$ strategies of finite volume discretization (including the DDFV method) were tested, see in particular [41]. Here we present some of the analogous results in the $3 D$ case, in three situations. More ample results and comparison to other methods will be presented in the $3 D$ benchmark [11, 42].

Let $\mathbb{P}^{\mathfrak{I}}$ denote the projection on the DDFV mesh $\mathfrak{T}$ (i.e., the components of $\mathbb{P}^{\mathfrak{T}} f$ are the mean values of $f \in L^{1}(\Omega)$ per primal and per dual volumes, cf. Remark 4.2(iii)). Let $\overrightarrow{\mathbb{P}}^{\mathfrak{z}}$ denote the projection on the diamond mesh $\mathfrak{D}$. The heterogeneity of the matrix $A$ is taken into account by using the diamond-wise projection $A^{\mathfrak{T}}:=\overrightarrow{\mathbb{P}}^{\mathfrak{T}} A$; similarly, we use $f^{\mathfrak{T}}=\mathbb{P}^{\mathfrak{T}} f$ as the discrete source term. Boundary data $\bar{u}$ are taken into account using the projection $\mathbb{P}^{\partial \mathfrak{T}}$ on boundary volumes: $\mathbb{P}^{\overline{\mathfrak{s}}}:=\left(\mathbb{P}^{\mathfrak{\tau}} \cdot, \mathbb{P}^{\partial \mathfrak{\tau}} \cdot\right)$. For a fully practical discretization of $A$ and $f$ (which are continuous in all our tests), for every element (recall that diamonds, primal volumes and dual volumes of a DDFV mesh are unions of elements, see Fig. 4) we take the mean value of the four vertices of the element.

Therefore, for the case of homogeneous boundary condition, given a DDFV mesh $\mathfrak{T}$ of $\Omega$ the method writes as:

$$
\text { find } u^{\overline{\mathfrak{T}}} \in \mathbb{R}_{0}^{\mathfrak{I}} \text { solution of }-\operatorname{div}^{\mathfrak{T}}\left[A^{\mathfrak{T}} \nabla^{\mathfrak{T}} u^{\overline{\mathfrak{T}}}\right]=f^{\mathfrak{T}} \text {. }
$$

More generally, the Dirichlet boundary conditions are prescribed at the centers of the boundary volumes $\partial \mathfrak{M}^{o}$ and $\partial \mathfrak{M}^{*}$ using $\mathbb{P}^{\partial \mathfrak{I}} \bar{u}$; in this case, $u^{\overline{\mathfrak{s}}}=\left(u^{\mathfrak{I}}, \mathbb{P}^{\partial \mathfrak{x}} \bar{u}\right)$ with $u^{\mathfrak{I}}$ unknown.

In each of the test cases described below, we start with a given mesh of the unit cube $\Omega$ (cartesian, tetrahedral, hexagonal, prismal) using it as the primal mesh for the DDFV construction; we choose the barycenters for the centers of these primal volumes and of their faces. It should be noticed that the dual mesh is never actually constructed, all the information is "read" from the primal mesh using in particular (6). The matrix and the source term of the linear system for the method are assembled per diamond.

Thanks to the duality property, one can easily prove that the resulting scheme is well-posed and convergent. It is also classical to prove an order $h$ convergence in the $H_{0}^{1}$ norm using e.g. 
the tools developed in $[9,20,3]$. Whenever the (uniform) discrete Poincare inequality can be justified, one derives an order $h$ convergence in the $L^{2}$ norm for the solution. This is the case if $l=3$ (i.e. the primal faces are triangles), but in general one can construct counterexamples to the discrete Poincaré inequality. Yet numerically, the value of $l$ does not seem important, and the order $h$ is a rather pessimistic one.

\section{- Measure of errors and convergence orders.}

To put the discrete and the exact solutions "at the same level", we use the projection $\mathbb{P}^{\mathfrak{s}} u_{e}$ of the exact solution and the associated discrete gradient reconstruction $\overrightarrow{\nabla^{\mathfrak{s}}} \mathbb{P}^{\overline{\mathfrak{s}}} u_{e}$, where $\mathbb{P}^{\overline{\mathfrak{s}}} \cdot=$

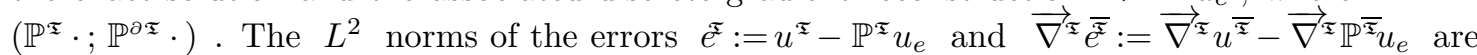
measured in terms of the scalar products $\mathbb{\|} \cdot, \cdot \|_{\Omega}$ on $\mathbb{R}^{\mathfrak{T}}$ and $\left\{[\cdot, \cdot\}_{\Omega}\right.$ on $\left(\mathbb{R}^{3}\right)^{\mathcal{P}}:$ the error indicators we use are relative errors defined, respectively, as



In all the tests, the convergence orders are reported wrt the number of unknowns (\#unkw).

\section{- Laplacian, uniform cartesian meshes and non-conformal checkerboard meshes}

In this case, we first take the uniform cartesian DDFV mesh of Section 2.3 (see Fig. 3) and we take the exact solution $u_{e}(x, y, z)=\sin (2 \pi x) \sin (2 \pi y) \sin (2 \pi z)$ of the homogeneous Dirichlet problem ( $f$ is calculated accordingly). It should be noted that the solution is smooth and both the mesh and the problem are symmetric wrt reflexions in each direction. Therefore error analysis based on cancellations in Taylor expansions (cf. [8] for the more difficult $p$-laplacian case in $2 D$ ) allows to prove the order $h^{2}$ convergence for the solution itself (measured in the $L^{2}$ norm) and for the discrete gradient (measured in the $L^{2}$ norm). The numerical results confirm these orders as optimal ones; indeed, the following table gives results and convergence orders:

\begin{tabular}{|c|c||c|c||c|c||}
\hline \#Cubes & \#unkw & Err L $^{2}$ & Order & ErrGrad L $^{2}$ & Order \\
\hline 6x6x6 & 341 & $0.207 \mathrm{E}+00$ & - & $0.226 \mathrm{E}+00$ & - \\
\hline 9x9x9 & 1241 & $0.830 \mathrm{E}-01$ & 2.118 & $0.936 \mathrm{E}-01$ & 2.048 \\
\hline $12 \times 12 \times 12$ & 3059 & $0.451 \mathrm{E}-01$ & 2.025 & $0.514 \mathrm{E}-01$ & 1.993 \\
\hline $18 \times 18 \times 18$ & 10745 & $0.196 \mathrm{E}-01$ & 1.992 & $0.225 \mathrm{E}-01$ & 1.977 \\
\hline $24 \times 24 \times 24$ & 25991 & $0.109 \mathrm{E}-01$ & 1.982 & $0.126 \mathrm{E}-01$ & 1.974 \\
\hline $32 \times 32 \times 32$ & 62559 & $0.612 \mathrm{E}-02$ & 1.981 & $0.704 \mathrm{E}-02$ & 1.976 \\
\hline
\end{tabular}

Then we consider the same problem discretized on non-conformal cartesian meshes; the meshes are made of cubes of size $h$ of which every second cube is refined into four cubes of size $h / 2$ (the so-called checkerboard meshes). The corresponding results are:

\begin{tabular}{|c|c||c|c||c|c||}
\hline \#Cubes & \#unkw & Err L $^{2}$ & Order & ErrGrad L & Order \\
\hline 59 & 703 & $0.433 \mathrm{E}+00$ & - & $0.462 \mathrm{E}+00$ & - \\
\hline 599 & 9835 & $0.172 \mathrm{E}+00$ & 1.198 & $0.172 \mathrm{E}+00$ & 1.278 \\
\hline 5423 & 101539 & $0.446 \mathrm{E}-01$ & 1.835 & $0.914 \mathrm{E}-01$ & 0.862 \\
\hline 46175 & 917395 & $0.110 \mathrm{E}-01$ & 1.966 & $0.460 \mathrm{E}-01$ & 0.962 \\
\hline 381119 & 7788403 & $0.273 \mathrm{E}-02$ & 1.977 & $0.231 \mathrm{E}-01$ & 0.979 \\
\hline
\end{tabular}

Here we clearly observe the superconvergence order $h^{2}$ for the solution in $L^{2}$ norm which would be much more difficult to justify theoretically; at the same time, the order of the discrete gradient convergence in the $L^{2}$ norm falls down to $h$.

- Mildly anisotropic permeability $A$, cartesian, tetrahedral and Kershaw meshes

In this test, the permeability matrix $A$ does not vary in space, but it is anisotropic:

$$
A \equiv\left(\begin{array}{ccc}
1 & 0.5 & 0 \\
0.5 & 1 & 0.5 \\
0 & 0.5 & 1
\end{array}\right)
$$


The exact solution is given by the formula $u_{e}(x, y, z)=1+\sin (\pi x) \sin \left(\pi\left(y+\frac{1}{2}\right)\right) \sin \left(\pi\left(z+\frac{1}{3}\right)\right)$, the data $\bar{u}$ and $f$ are calculated accordingly. We use three different kinds of primal meshes. Firstly, on the same cartesian meshes as in the previous case (see Section 2.3 and Fig. 3) we get the following results:

\begin{tabular}{|c||c|c||c|c||}
\hline \#Cubes & Err L $^{2}$ & Order & ErrGrad L $^{2}$ & Order \\
\hline $6 \times 6 \times 6$ & $0.742 \mathrm{E}-02$ & - & $0.376 \mathrm{E}-01$ & - \\
\hline 9x9x9 & $0.332 \mathrm{E}-02$ & 1.867 & $0.196 \mathrm{E}-01$ & 1.511 \\
\hline $12 \times 12 \times 12$ & $0.187 \mathrm{E}-02$ & 1.899 & $0.125 \mathrm{E}-01$ & 1.505 \\
\hline $18 \times 18 \times 18$ & $0.837 \mathrm{E}-03$ & 1.927 & $0.666 \mathrm{E}-02$ & 1.501 \\
\hline $24 \times 24 \times 24$ & $0.471 \mathrm{E}-03$ & 1.948 & $0.428 \mathrm{E}-02$ & 1.499 \\
\hline $32 \times 32 \times 32$ & $0.265 \mathrm{E}-03$ & 1.962 & $0.276 \mathrm{E}-02$ & 1.498 \\
\hline
\end{tabular}

Here, the $h^{2}$ convergence for the gradient is lost and $h^{3 / 2}$ convergence is observed, cf. [14] (clearly, the anisotropy the permeability matrix $A$ destroys some of the symmetries that led to superconvergence in the case of laplacian). Yet an almost $h^{2}$ convergence in $L^{2}$ for the solution itself is still observed.
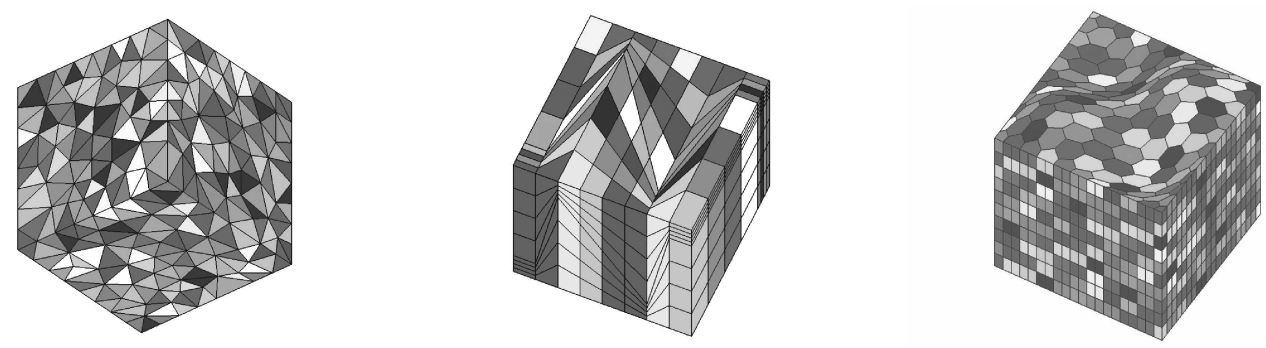

Figure 5. Meshes (primal): tetrahedral mesh on the left, Kershaw mesh at the center, and prismal mesh on the right

Secondly, we use tetrahedral primal meshes (see Fig. 5, left). They do not lead to the DelaunayVoronoï DDFV meshes of Example 4.3, because of the barycentric choice for the cell and face centers; cf. Hermeline [46] for the choice of circumcenters, on slightly different test cases. Actually, in the below test we have used tetrahedral meshes that do not respect the Delaunay property. Let us remind that our construction does not impose any specific choice for cell and face centers; only the edge centers must be fixed to be the middlepoints. The barycentric choice of cell and face centers leads to rather regular dual meshes. Tetrahedral mesh corresponds to $l=3$, which is the simple case of the gradient reconstruction formulas that can be understood as straightforward affine interpolation in $K L$. On the corresponding DDFV meshes, we get the following results:

\begin{tabular}{|c|c||c|c||c|c||}
\hline \#Tetras & \#unkw & Err L $^{2}$ & Order & ErrGrad L $^{2}$ & Order \\
\hline 2003 & 2187 & $0.539 \mathrm{E}-02$ & - & $0.654 \mathrm{E}-01$ & - \\
\hline 3898 & 4301 & $0.331 \mathrm{E}-02$ & 2.165 & $0.488 \mathrm{E}-01$ & 1.297 \\
\hline 7711 & 8584 & $0.206 \mathrm{E}-02$ & 2.069 & $0.381 \mathrm{E}-01$ & 1.077 \\
\hline 15266 & 17102 & $0.135 \mathrm{E}-02$ & 1.841 & $0.301 \mathrm{E}-01$ & 1.018 \\
\hline 30480 & 34343 & $0.846 \mathrm{E}-03$ & 1.998 & $0.240 \mathrm{E}-01$ & 0.973 \\
\hline 61052 & 69160 & $0.539 \mathrm{E}-03$ & 1.934 & $0.190 \mathrm{E}-01$ & 1.012 \\
\hline
\end{tabular}

One sees that here, the convergence order for the solution gradient falls down to $h$, while superconvergence is still observed for the $L^{2}$ norm of the solution itself.

Thirdly, we use the so-called Kershaw meshes (see Fig. 5, center) with hexahedral faces ${ }^{7}$. In this case, $l$ takes the value 4 ; here (5) is the (non-evident) choice of order one-consistent discrete

\footnotetext{
7 these meshes, generated by K. Lipnikov, are proposed in the 3D benchmark [42] (see http://www.latp.univ-mrs.fr/latp_numerique/?q=node/11) and can be visualized at the same Web location
} 
gradient that leads to discrete duality, see Remark 5.4 (we have $l=4$ also for the case of uniform cartesian meshes, but the symmetry of uniform meshes makes the gradient reconstruction formula (8) easy to guess). Starting from the Kershaw primal meshes, the tests provide the following table:

\begin{tabular}{|c|c||c|c||c|c||}
\hline \#Hexs & \#unkw & Err L $^{2}$ & Order & ErrGrad L $^{2}$ & Order \\
\hline $8 \times 8 \times 8$ & 855 & $0.501 \mathrm{E}-01$ & - & $0.484 \mathrm{E}+00$ & - \\
\hline $16 \times 16 \times 16$ & 7471 & $0.156 \mathrm{E}-01$ & 1.611 & $0.209 \mathrm{E}+00$ & 1.160 \\
\hline $32 \times 32 \times 32$ & 62559 & $0.392 \mathrm{E}-02$ & 1.954 & $0.677 \mathrm{E}-01$ & 1.594 \\
\hline $64 \times 64 \times 64$ & 512191 & $0.101 \mathrm{E}-02$ & 1.936 & $0.223 \mathrm{E}-01$ & 1.585 \\
\hline
\end{tabular}

Also here a convergence order slightly below $h^{2}$ for the solution itself is observed; order $h^{3 / 2}$ appears for the gradient, making the results similar to those on cartesian meshes.

\section{- Heterogeneous permeability $A$, prismal meshes}

Here, distorted prismal meshes (see Fig. 5, right) with rather deformed faces are used ${ }^{8}$ The permeability matrix $A$ is heterogeneous, given by

$$
A(x, y, z)=\left(\begin{array}{ccc}
y^{2}+z^{2}+1 & -x y & -x z \\
-x y & x^{2}+z^{2}+1 & -y z \\
-x z & -y z & x^{2}+y^{2}+1
\end{array}\right),
$$

and the exact solution of the problem is, $u_{e}(x, y, z)=x^{3} y^{2} z+x \sin (2 \pi x z) \sin (2 \pi x y) \sin (2 \pi z)$ with the ad hoc source and boundary values $f, \bar{u}$. Here, This test was proposed in [12], see also [53]. Starting from these prismal meshes, we obtain the following table:

\begin{tabular}{|c|c||c|c||c|c||}
\hline \#Prisms & \#unkw & Err L $^{2}$ & Order & ErrGrad L $^{2}$ & Order \\
\hline 1210 & 3010 & $0.467 \mathrm{E}-01$ & - & $0.711 \mathrm{E}-01$ & - \\
\hline 8820 & 24020 & $0.123 \mathrm{E}-01$ & 1.931 & $0.224 \mathrm{E}-01$ & 1.667 \\
\hline 28830 & 81030 & $0.554 \mathrm{E}-02$ & 1.960 & $0.116 \mathrm{E}-01$ & 1.634 \\
\hline 67240 & 192040 & $0.314 \mathrm{E}-02$ & 1.973 & $0.728 \mathrm{E}-02$ & 1.607 \\
\hline
\end{tabular}

Here we see that the quality of approximation is comparable to the one of the previous test on cartesian and on Kershaw meshes; this makes us think that the scheme supports well mild heterogeneity of the diffusion tensor (at least for smooth enough dependence on the space variable and smooth solution), Moreover, elevated number of face vertices (here, we have up to $l=6$ vertices per face) does not alter the scheme's behaviour.

In conclusion, good convergence properties of the scheme are observed on each of the preceding meshes, both for anisotropic and for heterogeneous diffusion problems. Superconvergence is observed for the laplacian on uniform cartesian mesh, and the other cases, $L^{2}$ convergence orders intermediate between $h^{3 / 2}$ and $h^{2}$ for the solution, and orders $h$ or $h^{3 / 2}$ for the gradient, are manifested. Elevated number $l$ of face vertices seems less damaging than poor shape regularity of the meshes.

Other properties of schemes are of importance, such as fulfillment or violation of the maximum principle. While maximum principle can be shown in some situations, specifically on orthogonal tetrahedral meshes for isotropic problems (see, e.g., [5],[3, Prop.4.3]), for most anisotropic or heterogeneous diffusion problems the maximum principle is, in general, false. In practice, this depends however on the stiffness of the solution, on the strength of anisotropy and heterogeneity, and on shape regularity of the meshes. The numerical observations presented in [11] show that over- and undershoots do not occur or they remain imperceptible, provided the anisotropy is a moderate one and the meshes are not strongly distorted.

\footnotetext{
$8_{\text {these meshes, generated by G. Manzini, were also proposed in the benchmark [42] }}$ (http://www.latp.univ-mrs.fr/latp_numerique/?q=node/11); in the primal faces $K \mid L$ we find two kinds of values, $l=4$ and $l=6$.
} 


\section{Appendix: A ReCOnStruCtion PROPERTy in the Plane}

Now we motivate the definition of the discrete gradient operator given in Section 2. Although Lemma 5.1 below is a purely two-dimensional property, it is convenient to use the 3D formalism. The subsequent notation corresponds to Figure 6; within this paragraph, the reader may ignore all "asterisk" superscripts.

Let $\Pi$ be a plane in $\mathbb{R}^{3}$ with a fixed unit normal vector $\vec{n}$, and $\sigma \subset \Pi$ be a polygon with $l$ vertices $(l \geq 3)$ denoted $x_{1}^{*}, \ldots, x_{l}^{*}$, numbered in the direct sense with respect to the orientation of $\Pi$ induced by $\vec{n}$. This means that for $i \in \llbracket 1, l \rrbracket, x_{i}^{*}$ and $x_{i+1}^{*}$ are neighbour vertices of the polygon $\sigma$, and $\left.<\vec{\nu}_{i, i+1}^{*}, \vec{e}_{i, i+1}^{*}, \vec{n}\right\rangle=1$; here $\vec{e}_{i, i+1}^{*}=\overrightarrow{x_{i}^{*} x_{i+1}^{*}} /\left\|x_{i}^{*} x_{i+1}^{*}\right\|$ and $\vec{\nu}_{i, i+1}^{*}$ is the unit normal vector to $\left[x_{i}^{*}, x_{i+1}^{*}\right]$ lying in $\Pi$ and pointing outside $\sigma$. Here and in the sequel, $x_{l+1}^{*}$ stands for $x_{1}^{*}$.

Further, introduce the barycenter (i.e., the middlepoint) $x_{i, i+1}^{*}$ of $\left[x_{i}^{*}, x_{i+1}^{*}\right]$. For a point $x_{\sigma}^{*} \in \Pi$ and $i \in \llbracket 1, l \rrbracket$ such that $x_{i, i+1}^{*} \neq x_{\sigma}^{*}$, denote $\vec{\tau}_{i, i+1}^{*}=\overrightarrow{x_{\sigma}^{*} x_{i, i+1}^{*}} /\left\|\overrightarrow{x_{\sigma}^{*} x_{i, i+1}^{*}}\right\|$ and $\vec{a}_{i, i+1}^{*}=\vec{n} \times \vec{\tau}_{i, i+1}^{*}$. In this way, for each $i \in \llbracket 1, l \rrbracket, \Pi$ is supplied with a couple of orthonormal bases $\left(\vec{\nu}_{i, i+1}^{*}, \vec{e}_{i, i+1}^{*}\right)$ and $\left(\vec{\tau}_{i, i+1}^{*}, \vec{a}_{i, i+1}^{*}\right)$, both oriented in the direct sense.

Finally, denote by $\alpha_{i, i+1}^{*}$ the angle between $\vec{\nu}_{i, i+1}^{*}$ and $\vec{\tau}_{i, i+1}^{*}$; this is also the angle between $\vec{e}_{i, i+1}^{*}$ and $\vec{a}_{i, i+1}^{*}$. Set

$$
\left.m_{i, i+1}=\frac{1}{2}\left\langle\vec{n}, \overrightarrow{x_{\sigma}^{*} x_{i, i+1}^{*}}, \overrightarrow{x_{i}^{*} x_{i+1}^{*}}\right\rangle \quad \text { ( we have } m_{i, i+1}=\frac{1}{2}\left\|\overrightarrow{x_{i}^{*} x_{i+1}^{*}}\right\|\left\|\overrightarrow{x_{\sigma}^{*} x_{i, i+1}^{*}}\right\| \cos \alpha_{i, i+1}^{*}\right)
$$

this is the (signed) area of the triangle $x_{i}^{*} x_{\sigma}^{*} x_{i+1}^{*}$. Denote the area of $\sigma$ by $m$; it is easily seen that $m=\sum_{i=1}^{l} m_{i, i+1}$. We also have $2 \frac{m_{i, i+1}}{\cos \alpha_{i, i+1}^{*}} \vec{a}_{i, i+1}^{*}=\left\|\overrightarrow{x_{i}^{*} x_{i+1}^{*}}\right\|\left[\vec{n} \times \overrightarrow{x_{\sigma}^{*} x_{i, i+1}^{*}}\right]$. In the case $m_{i, i+1}=0$ (this happens, e.g., if $x_{i, i+1}^{*}=x_{\sigma}^{*}$ ), the left-hand side of the above expression is meant to be zero.


FiguRE 6. 2D Reconstruction property (zoom on a primal interface)

Lemma 5.1. With the notation above, for all vector $\vec{r}$ parallel to $\Pi$ we have

$$
\vec{r}=\frac{2}{m} \sum_{i=1}^{l} \frac{m_{i, i+1}}{\cos \alpha_{i, i+1}^{*}}\left(\vec{r} \cdot \vec{e}_{i, i+1}^{*}\right) \vec{a}_{i, i+1}^{*}=\frac{1}{m} \sum_{i=1}^{l}\left(\vec{r} \cdot \overrightarrow{x_{i}^{*} x_{i+1}^{*}}\right)\left[\vec{n} \times \overrightarrow{x_{\sigma}^{*} x_{i, i+1}^{*}}\right] .
$$

Note that, if $\sigma$ admits a circumscribed circle and $x_{\sigma}^{*}$ is chosen to be its center, then $\vec{\tau}_{i, i+1}^{*}$ is parallel to $\vec{\nu}_{i, i+1}^{*}$; in this case (23) reduces to the reconstruction property used in [5].

Proof : Consider $\vec{r}$ parallel to $\Pi$. Upon rotating axes, assume $\Pi$ is the $X O Y$ plane, and forgot temporarily the third coordinate axis. Define the function $u: x \in \Pi \mapsto \vec{r} \cdot \overrightarrow{x_{\sigma}^{*} x}$. We have 
$\left(r_{1}, r_{2}\right)=\vec{r}=\nabla u=\left(\partial_{1} u, \partial_{2} u\right)$, and the Green-Gauss integration-by-parts formula yields

$$
m r_{1}=\int_{\sigma} \partial_{1} u=\int_{\sigma} \operatorname{div} \overrightarrow{(u, 0)}=\int_{\partial \sigma} \overrightarrow{(u, 0)} \cdot \vec{\nu}=\int_{\partial \sigma} u \nu_{1}
$$

where $\nu=\left(\nu_{1}, \nu_{2}\right)$ is the exterior unit normal vector to $\partial \sigma$. Analogous calculation for the second component yields $m r_{2}=\int_{\partial \sigma} u \nu_{2}$. Splitting the integral over $\partial \sigma$ into $l$ integrals over edges $\left[x_{i}^{*}, x_{i+1}^{*}\right]$, using the definition of $u$ and using the barycenter $x_{i, i+1}^{*}$ of $\left[x_{i}^{*}, x_{i+1}^{*}\right]$ to evaluate the integral of the affine function $u$, we get

$$
\vec{r}=\frac{1}{m} \sum_{i=1}^{l}\left\|\overrightarrow{x_{i}^{*} x_{i+1}^{*}}\right\|\left(\vec{r} \cdot \overrightarrow{x_{\sigma}^{*} x_{i, i+1}^{*}}\right) \vec{\nu}_{i, i+1}^{*}=\frac{2}{m} \sum_{i=1}^{l} \frac{m_{i, i+1}}{\cos \alpha_{i, i+1}^{*}}\left(\vec{r} \cdot \vec{\tau}_{i, i+1}^{*}\right) \vec{\nu}_{i, i+1}^{*}
$$

for all vector $\vec{r}$ parallel to $\Pi$ (formula (22) is used for the last equality).

Furthermore, for all $i \in \llbracket 1, l \rrbracket$, we have $\cos \alpha_{i, i+1}^{*} \vec{r}=\left(\vec{r} \cdot \vec{\tau}_{i, i+1}^{*}\right) \vec{\nu}_{i, i+1}^{*}+\left(\vec{r} \cdot \vec{e}_{i, i+1}^{*}\right) \vec{a}_{i, i+1}^{*}$. For the proof, it suffices to take the scalar product by $\vec{\tau}_{i, i+1}^{*}$, and then by $\vec{e}_{i, i+1}^{*}$, of the both sides of the relation.

Therefore

$$
2 \vec{r}=\frac{2}{m} \sum_{i=1}^{l} \frac{m_{i, i+1}}{\cos \alpha_{i, i+1}^{*}}\left(\left(\vec{r} \cdot \vec{\tau}_{i, i+1}^{*}\right) \vec{\nu}_{i, i+1}^{*}+\left(\vec{r} \cdot \vec{e}_{i, i+1}^{*}\right) \vec{a}_{i, i+1}^{*}\right) .
$$

Subtracting (24) from (25) term by term, using the third coordinate axis to represent $\vec{a}_{i, i+1}^{*}$ by a vector product, we deduce (23).

Corollary 5.2. With the above notation, take $\left(w_{i}^{*}\right)_{i=1}^{l} \subset \mathbb{R}, w_{l+1}^{*}:=w_{1}^{*}$. Consider the expression

$$
\frac{1}{\sum_{i=1}^{l} m_{i, i+1}} \sum_{i=1}^{l}\left(w_{i+1}^{*}-w_{i}^{*}\right)\left[\vec{n} \times \overrightarrow{x_{\sigma}^{*} x_{i, i+1}^{*}}\right]
$$

In the case $w_{i}^{*}$ are the values of an affine function $w$ at the vertices $x_{i}^{*}$ of the polygon $\sigma$, expression (26) gives the projection $\operatorname{Proj}^{*}(\nabla w)$ of $\nabla w$ on the plane $\Pi$.

ProOF : It is sufficient to note that $\operatorname{Proj}^{*}(\nabla w) \cdot \overrightarrow{x_{i}^{*} x_{i+1}^{*}}=\nabla w \cdot \overrightarrow{x_{i}^{*} x_{i+1}^{*}}=w_{i+1}^{*}-w_{i}^{*}$, and to substitute $m=\sum_{i=1}^{l} m_{i, i+1}$ into the right-hand side of (23).

Remark 5.3. Notice that Lemma 5.1 and Corollary 5.2 hold for all choice of $x_{\sigma}^{*}$ in the plane $\Pi$; the restriction $x_{\sigma}^{*} \in \sigma$ is not necessary. This restriction is equivalent to the positivity of $m_{i, i+1}$ for all $i$.

Remark 5.4. If $l=3$, then (26) is just a non-evident way, suitable for the proof of Proposition 3.2, to express the gradient of the unique function obtained by the three-point affine interpolation. For the practical computation of the discrete gradient, one can take any convenient formula expressing this three-point interpolation.

Let us point out that, unless $l=3$, formula (26) determines one among infinitely many linear forms in $\left(w_{i}^{*}\right)_{i=1}^{l}$ which share the property of Corollary 5.2. The choice of (26) gives a particular role to a given point $x_{\sigma}^{*}$.

We guess that the affine interpolation formula (26) is well known, but to the best of our knowledge, it was not yet exploited in the context of finite volume schemes. It can be used for different vertex-centered finite volume schemes in the same way as the gradient reconstruction formula shown in [32, Lemma 6.1], also known as the "magical formula", is used for the cellcentered schemes (see e.g. formula (23) in [35]). 
B. ANDREIANOV, M. BENDAHMANE, F. HUBERT, AND S. KRELL

\section{REFERENCES}

[1] I. Aavatsmark, T. Barkve, $\varnothing$. Bœ, and T. Mannseth. Discretization on unstructured grids for inhomogeneous, anisotropic media. Part I: derivation of the methods. SIAM J.Sci.Comp. 19(5):1700-1716, 1998.

[2] M. Afif and B. Amaziane. Convergence of finite volume schemes for a degenerate convection-diffusion equation arising in flows in porous media, Comput. Methods Appl. Mech. Eng. 191(46):5265-5286, 2002.

[3] B. Andreianov, M. Bendahmane and F. Hubert. On 3D DDFV discretization of gradient and divergence operators. II. Discrete functional analysis tools and applications to degenerate parabolic problems. Preprint HAL 2011, http://hal.archives-ouvertes.fr/hal-00567342

[4] B. Andreianov, M. Bendahmane and K.H. Karlsen. A gradient reconstruction formula for finite volume schemes and discrete duality. In R. Eymard and J.-M. Hérard, editors, Finite Volume For Complex Applications, Problems And Perspectives. 5th International Conference, 161-168. London (UK) Wiley, 2008.

[5] B. Andreianov, M. Bendahmane, and K.H. Karlsen. Discrete duality finite volume schemes for doubly nonlinear degenerate hyperbolic-parabolic equations. J. Hyp. Diff. Equ. 7(1):1-67, 2010.

[6] B. Andreianov, M. Bendahmane, K.H. Karlsen and Ch. Pierre. Convergence of Discrete Duality Finite Volume schemes for the macroscopic bidomain model of the heart electric activity. Netw. Heter. Media, accepted. Available as reprint HAL 2010, http://hal.archives-ouvertes.fr/hal-00526047

[7] B. Andreianov, F. Boyer, and F. Hubert. Finite volume schemes for the $p$-Laplacian on Cartesian meshes. M2AN Math. Model. Numer. Anal. 38(6):931-959, 2004.

[8] B. Andreianov, F. Boyer and F. Hubert. On finite volume approximation of regular solutions of the $p$-laplacian. IMA J.Numer. Anal., 26(3):472-502, 2006.

[9] B. Andreianov, F. Boyer and F. Hubert. Discrete duality finite volume schemes for Leray-Lions type elliptic problems on general 2D meshes. Num. Meth. PDE, 23(1):145-195, 2007.

[10] B. Andreianov, M. Gutnic and P. Wittbold. Convergence of finite volume approximations for a nonlinear elliptic-parabolic problem: a "continuous" approach. SIAM J. Numer. Anal. 42(1):228-251, 2004.

[11] B. Andreianov, F. Hubert and S. Krell. Benchmark 3D: a version of the DDFV scheme with cell/vertex unknowns on general meshes. In Proceedings of Finite Volumes for Complex Applications VI in Prague, Springer, 2011, to appear.

[12] F. Brezzi, A. Buffa and K. Lipnikov Mimetic finite differences for elliptic problems ESAIM: M2AN 43(2):277295,2009 .

[13] F. Boyer and F. Hubert. Finite volume method for 2D linear and nonlinear elliptic problems with discontinuities. SIAM J. Num. Anal. 46(6):3032-3070, 2008.

[14] F. Boyer and F. Hubert. Benchmark for Anisotropic Problems. The DDFV "discrete duality finite volumes" and m-DDFV schemes, in R. Eymard and J. M. Herard, eds., Proceedings of the 5th international symposium on Finite Volumes for Complex Applications. Hermès, 2008.

[15] F. Boyer, F. Hubert and S. Krell. Non-overlapping Schwarz algorithm for solving 2D-mDDFV schemes. IMA J. Num. Anal. 30(4):1062-1100, 2010.

[16] F. Brezzi, K. Lipnikov and M. Shashkov. Convergence of mimetic finite difference method for diffusion problems on polyhedral meshes. SIAM J. Numer. Anal. 43(5):1872-1896, 2005.

[17] F. Brezzi, K. Lipnikov and V. Simoncini. A family of mimetic finite difference methods on polygonal and polyhedral meshes. Math. Models Methods Appl. Sci. 15(10):1533-1551, 2005.

[18] S. Corsaro, K. Mikula, A. Sarti and F. Sgallari. Semi-implicit co-volume method in 3D image segmentation. SIAM J. Sci. Comput., 28(6):2248-2265, 2006.

[19] Y. Coudière and F. Hubert. A 3D Discrete Duality Finite Volume method for nonlinear elliptic equations. In: A. Handlovičovà, P. Frolkovič, K. Mikula, D. Ševčovič (Eds.), Proceedings of Algoritmy 2009, pp. 51-60, 2009.

[20] Y. Coudière and F. Hubert. A 3D discrete duality finite volume method for nonlinear elliptic equation. HAL preprint 2010, http://hal.archives-ouvertes.fr/hal-00456837

[21] Y. Coudière, F. Hubert and G. Manzini. Benchmark 3D: CeVeFE-DDFV, a discrete duality scheme with cell/vertex/face+edge unknowns. In Proceedings of Finite Volumes for Complex Applications VI in Prague, Springer, 2011, to appear.

[22] Y. Coudière and G. Manzini. The Discrete Duality Finite Volume Method for Convection-diffusion Problems SIAM J. Numer. Anal. Volume 47(6):4163-4192, 2010.

[23] Y. Coudière and Ch. Pierre. Benchmark 3D: CeVe-DDFV, a discrete duality scheme with cell/vertex unknowns. In Proceedings of Finite Volumes for Complex Applications VI in Prague, Springer, 2011, to appear.

[24] Y. Coudière, Ch. Pierre, and R. Turpault. Solving the fully coupled heart and torso problems of electrocardiology with a 3D discrete duality finite volume method. submitted for publication. Preprint HAL 2009, http://hal.archives-ouvertes.fr/hal-00016825

[25] Y. Coudière, Ch. Pierre, O. Rousseau and R. Turpault. 2D/3D discrete duality finite volume scheme (DDFV) applied to ECG simulation. In R. Eymard and J.-M. Hérard, editors, Finite Volume For Complex Applications, Problems And Perspectives. 5th International Conference, 313-320. London (UK) Wiley, 2008. 
[26] Y. Coudière, Ch. Pierre, O. Rousseau and R. Turpault. 2D/3D discrete duality finite volume scheme. Application to ECG simulation. Int. J. Finite Vol. 6(1):1-24, 2009.

[27] Y. Coudière, J.-P. Vila and Ph. Villedieu. Convergence rate of a finite volume scheme for a two-dimensional convection-diffusion problem. M2AN Math. Model. Numer. Anal. 33(3):493-516, 1999.

[28] S. Delcourte. Développement de méthodes de volumes finis pour la mécanique de fluides. (French) Ph.D. thesis, Univ. Paul Sabatier, Toulouse, 2007.

[29] K. Domelevo, S. Delcourte and P. Omnes. Discrete-duality finite volume method for second order elliptic equations, in: F. Benkhaldoun, D. Ouazar, S. Raghay (Eds.) Finite Volumes for Complex Applications pp.447-458, Hermès, 2005.

[30] K. Domelevo and P. Omnès. A finite volume method for the Laplace equation on almost arbitrary twodimensional grids. M2AN Math. Model. Numer. Anal. 39(6):1203-1249, 2005.

[31] J. Droniou. Finite volume approximations for fully nonlinear elliptic equations in divergence form. M2AN Math. Model. Numer. Anal. 40(6):1069-1100, 2006.

[32] R. Eymard and J. Droniou. A mixed finite volume scheme for anisotropic diffusion problems on any grid. Numer. Math. 105(1):35-71, 2006.

[33] R. Eymard, T. Gallouët, and R. Herbin. A cell-centered finite-volume approximation for anisotropic diffusion operators on unstructured meshes in any space dimension. IMA J. Numer. Anal. 26(2):326-353, 2006.

[34] R. Eymard, T. Gallouët, and R. Herbin. A new finite volume scheme for anisotropic diffusion problems on general grids: convergence analysis. C. R. Math. Acad. Sci. Paris 344(6):403-406, 2006.

[35] R. Eymard, T. Gallouët, and R. Herbin. Discretisation of heterogeneous and anisotropic diffusion problems on general non-conforming meshes. SUSHI: a scheme using stabilisation and hybrid interfaces, IMA J. Numer. Anal. 30(4)1009-1043, 2010.

[36] R. Eymard, T. Gallouët, and R. Herbin. Cell centerd discretisation of non linear elliptic problems on general multidimensional polyhedral grids. J. Numer. Math. 17(3):173-193, 2009.

[37] R. Eymard and R. Herbin. A new collocated FV scheme for the incompressible Navier-Stokes equations on general non-matching grids. C. R. Math. Acad. Sci. Paris, 344(10):659-662, 2007.

[38] R. Eymard, R. Herbin and C. Guichard. Small-stencil 3D schemes for diffusive flows in porous media. HAL preprint 2011, http://hal.archives-ouvertes.fr/hal-00542667

[39] A. Handlovičová and K. Mikula. Stability and consistency of the semi-implicit co-volume scheme for regularized mean curvature flow equation in level set formulation. Appl. Math. 53(2):105-129, 2008.

[40] A. Handlovičová, K. Mikula, and F. Sgallari. Semi-implicit complementary volume scheme for solving level set like equations in image processing and curve evolution, Numer. Math., 93(4):675-695, 2003.

[41] R. Herbin and F. Hubert. Benchmark on discretization schemes for anisotropic diffusion problems on general grids. In R. Eymard and J.-M. Hérard, editors, Finite Volume For Complex Applications, Problems And Perspectives. 5th International Conference, 659-692. London (UK) Wiley, 2008.

[42] R. Herbin and F. Hubert. Benchmark on discretization schemes for anisotropic diffusion problems on general $3 D$ grids. In Proceedings of Finite Volumes for Complex Applications VI in Prague, Springer, 2011, to appear.

[43] F. Hermeline. Une méthode de volumes finis pour les équations elliptiques du second ordre. (French) $C . R$. Math. Acad. Sci. Paris 326(12):1433-1436, 1998.

[44] F. Hermeline. A finite volume method for the approximation of diffusion operators on distorted meshes. $J$. Comput. Phys. 160(2):481-499, 2000.

[45] F. Hermeline. A finite volume method for solving Maxwell equations in inhomogeneous media on arbitrary meshes. C. R. Math. Acad. Sci. Paris 339(12):893-898, 2004.

[46] F. Hermeline. Approximation of $2 \mathrm{D}$ and $3 \mathrm{D}$ diffusion operators with variable full-tensor coefficients on arbitrary meshes. Comput. Methods Appl. Mech. Engrg., 196(21-24):2497-2526, 2007.

[47] F. Hermeline. A finite volume method for approximating 3D diffusion operators on general meshes. J. Comput. Phys. 228(16), 5763-5786, 2009.

[48] X.H. Hu and R.A. Nicolaides. Covolume techniques for anisotropic media. Numer. Math., 61:215-234, 1992.

[49] S. Krell. Schémas Volumes Finis en mécanique des fluides complexes. (French) Ph.D. Thesis, Univ. de Provence, Marseilles, 2010.

[50] S. Krell. Stabilized DDFV schemes for Stokes problem with variable viscosity on general 2D meshes. Num. Meth. PDEs 2010, http://dx.doi.org/10.1002/num.20603

[51] S. Krell and G. Manzini. The Discrete Duality Finite Volume method for the Stokes equations on 3D polyhedral meshes. HAL preprint 2010, http://hal.archives-ouvertes.fr/hal-00448465/

[52] Ch. Le Potier. Schéma volumes finis monotone pour des opérateurs de diffusion fortement anisotropes sur des maillages de triangles non structurés, C. R. Math. Acad. Sci. Paris, 341(12):787-792, 2005.

[53] K. Lipnikov, M. Shashkov, D. Svyatskiy and Yu. Vassilevski. Monotone finite volume schemes for diffusion equations on unstructured triangular and shape-regular polygonal meshes. J. Comput. Physics, 227(1):492-512, 2007. 
[54] K. Mikula, A. Sarti and F. Sgallari. Co-volume level set method in subjective surface based medical image segmentation. In: Handbook of Medical Image Analysis: Segmentation and Registration Models (J.Suri et al., Eds.) pp. 583-626, Springer, New York, 2005.

[55] K. Mikula, A. Sarti and F. Sgallari. Co-volume method for Riemannian mean curvature flow in subjective surfaces multiscale segmentation. Comput. Vis. Sci. 9(1):23-31, 2006.

[56] R.A. Nicolaides. Direct discretization of planar div-curl problems. SIAM J. Numer. Anal., 29:32-56, 1992.

[57] Ch. Pierre. Modélisation et simulation de l'activité électrique du coeur dans le thorax, analyse numérique et méthodes de volumes finis. (French) Ph.D. Thesis, Université de Nantes, 2005.

[58] Ch. Pierre. Private communication.

[59] N. J. Walkington. Algorithms for computing motion by mean curvature. SIAM J. Numer. Anal. 33(6):2215$2238,1996$.

Boris Andreianov, Laboratoire de Mathématiques de Besançon CNRS UMR6623, Université de FrancheComté, 16 Route DE Gray, 25030 Besançon, France; boris.andreianov@univ-fcomte.fr

Mostafa Bendahmane, Institut de Mathématiques de Bordeaux, Université Victor Segalen, 33076

Bordeaux Cedex, France mostafa.bendahmane@u-bordeaux2.fr

Florence Hubert, LATP, Université de Provence, 39 rue F. Joliot-Curie, 13453 Marseille Cedex 13, FRANCE; fhubert@cmi.univ-mrs.fr

Stella Krell, inRia lille Nord Europe, 40 avenue Halley, 59650 Villeneuve d'Ascq Cedex, France; krell@inria.fr 